\title{
On the influence of local fluctuations in volume-fraction of constituents on the effective properties of nonlinear composites. Application to porous materials
}

\author{
M. Gărăjeu ${ }^{1, *}$, P. Suquet \\ L.M.A./C.N.R.S., 31 Chemin Joseph Aiguier, 13402 Marseille Cedex 20, France
}

\begin{abstract}
Composite materials often exhibit local fluctuations in the volume fraction of their individual constituents. This paper studies the influence of such small fluctuations on the effective properties of composites. A general asymptotic expansion of these properties in terms of powers of the amplitude of the fluctuations is given first. Then, this general result is applied to porous materials.

As is well-known, the effective yield surface of ductile voided materials is accurately described by Gurson's criterion. Suitable extensions for viscoplastic solids have also been proposed. The question adressed in the present study pertains to nonuniform distributions of voids in a typical volume element or in other words to the presence of matrix-rich and pore-rich zones in the material. It is shown numerically and analytically that such deviations from a uniform distribution result in a weakening of the macroscopic carrying capacity of the material.
\end{abstract}

Key words: Inhomogeneous material, Viscoplastic material, Homogenization, Field fluctuations, Porous material, Gurson criterion

\section{Introduction}

The macroscopic properties of heterogeneous materials, such as composites or porous materials, depend in an essential manner on their microstructure.

\footnotetext{
* Corresponding author.

Email addresses: mihai.garajeu@univ.u-3mrs.fr (M. Gărăjeu), suquet@lma.cnrs-mrs.fr (P. Suquet).

1 Present address: Aix-Marseille Univ, EA 2596, Marseille, F-13397, France
} 
One of the most important descriptor of this microstructure is the volume fraction of the individual constituents. While the volume fraction of the phases is macroscopically constant, its fluctuations at a local level are essential in understanding a number of problems, including scattering by heterogeneities or fracture of composite materials.

For instance, random, but nonuniform, distributions of particles arise during processing of metal-matrix composites. Clustering of particles may play a role in the stress concentrations which eventually lead to breakage of particles and fracture of the composite. Clustering effects in particle-reinforced composites have been simulated numerically by Segurado et al. (2003). They found that the effect of reinforcement clustering is weak in the linear elastic regime but more pronounced, although limited, in the nonlinear plastic regime. They also found a dramatic increase in the number of particles broken in the clusters. This increase is due to the modification of the local fields due to clustering.

Similar effects have been observed in voided materials. For instance, Rossoll et al. (2002) observed in an experimental study on nuclear steels that clusters of precipitates constitute, after debonding, initiation sites for void growth and coalescence. These clusters are characterized by a local volume-fraction which is higher than the average volume-fraction in the material. Motivated by this observation, Bilger et al. (2005) investigated void clustering effects in ideally plastic materials. Their study confirms that while void clustering has almost no effect on the linear elastic properties of voided materials, it has a noticeable effect on their plastic properties. Considering three different types of void distributions, random distribution without clusters, distributions with disconnected clusters and distributions with connected clusters, they found that the flow surface of materials containing a random distribution of voids is a strict outer bound for the flow surface of materials containing disconnected clusters of voids which itself is an outer bound for materials in which the void clusters are connected. The degeneracy of the ideally plastic model explains probably why the influence observed by Bilger et al. (2005) is larger than that observed by Segurado et al. (2003) whose study concerned a matrix with significant isotropic hardening. Bilger et al. noticed in addition a strong influence of the stress state on the clustering effect. More specifically the effect of clusters was larger for hydrostatic loadings than for pure shear loadings. To conclude, there is a indisputable body of results, both experimental or computational, showing the role of local volume fraction fluctuations on the effective properties of heterogeneous materials. This effect, enhanced by the material nonlinearity, is probably more pronounced for porous materials than for reinforced composites and depends on the loading.

The aim of the present study is to assess this problem by analytical means. Clustering effects can be seen as a local variation in the volume fraction of the constituents inside the composite materials, the clusters of one phase being 
zones of higher volume-fraction of this particular phase. Therefore the question of clustering effects is transformed into the following one: how do fluctuations in the volume fraction of constituents affect the effective properties of composites? Do they strengthen or weaken the material? What is the role played by the material nonlinearity? How does this influence depend on the loading conditions?

This paper is organized as follows. Section 2 presents the class of composites under consideration in this study. They are characterized by three separate length-scales, called the micro, meso and macro-scale. The volume fraction of the constituents is uniform at the macro-scale but has fluctuations at the mesoscale. The first theoretical result of the paper is given in section 3 , where an asymptotic expansion of the macroscopic energy of the composite in powers of the volume-fraction fluctuations is given. It is shown in particular that the difference between the energy with and without fluctuations is, to second-order, proportional to the standard deviation of the fluctuations. This expansion is applied in section 4 to porous nonlinear materials, for which the effect of clustering is expected to be important. The effective properties of porous materials containing an isotropic distribution of spherical pores with uniform porosity is well described by Gurson's model (Gurson, 1977) (see section 2.3 for details). The present study will also make use of an extension of Gurson's model to viscous materials, based on the same choice of velocity fields as in the original work by Gurson. This extension, proposed by Leblond et al. (1994) is recalled in section 2.3. The main finding of section 4 is that, for materials whose effective properties are well described by Gurson's model or by its extension to viscous materials (roughly speaking, these materials have a microstructure close to an ideal assemblage of hollow spheres), local fluctuations in porosity always result in a weakening of the material properties. This observation, first made by means of numerical simulations, is confirmed by analytical results for most loading conditions. A very strong influence of the nonlinearity is found. In the ideally plastic limit the corrective term can even become infinite for deformation conditions which are investigated in detail in section 5. Finally section 6 investigates the effect of volume-fraction fluctuations on the flow surface of porous materials, rather than on their effective potential.

Throughout the text, vectors and second-order tensors will be denoted with boldface letters, whereas fourth-order tensors will be denoted by barred letters. In this connection, the various types of products will be denoted by dots (e.g., $\left.\boldsymbol{u} . \boldsymbol{v}=u_{i} v_{i},(\mathbb{L}: \boldsymbol{\varepsilon})_{i j}=L_{i j k h} \varepsilon_{k h}\right)$. 


\section{Problem setting}

\subsection{Clusters, volume-fraction fluctuations and three-scale composites}

For definiteness, the composites considered in this study are two-phase materials. For simplicity, they are also assumed to be composed of isotropic phases arranged isotropically (both assumptions can be removed). The existence of clusters of one phase is modelled as fluctuations in the local volume fraction $f$ of one of the phases, say phase 1 , about a mean value $f_{0}$.

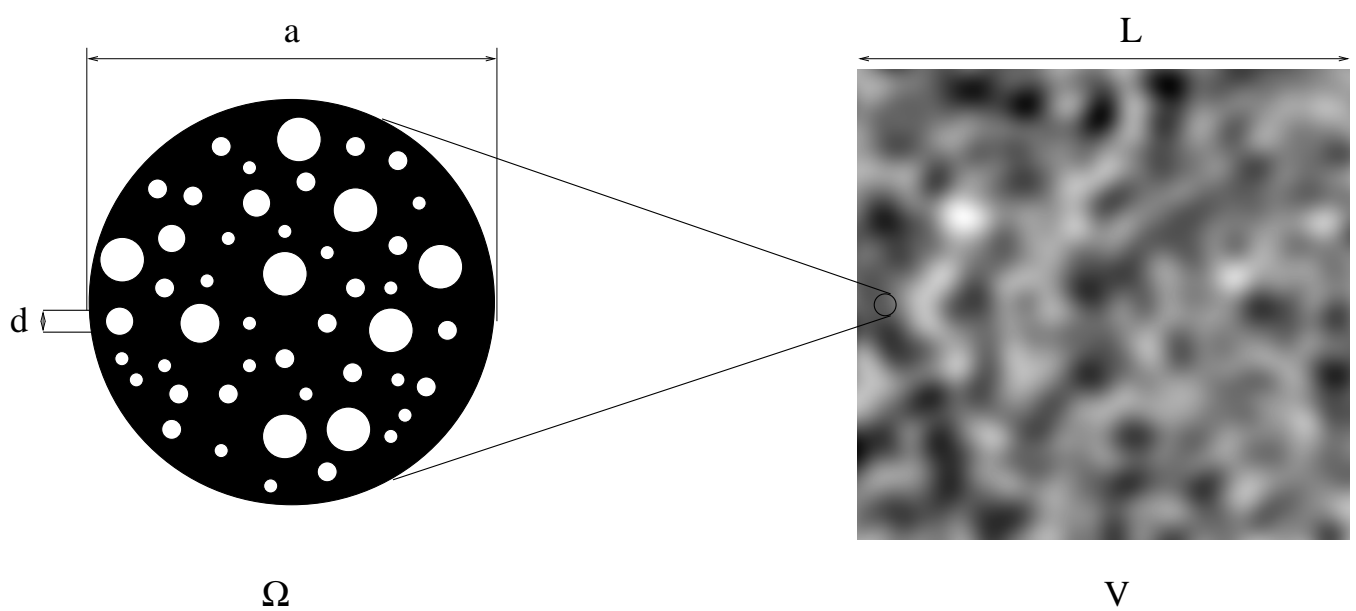

Fig. 1. Three-scale composite. Left: representative volume element $\Omega$ seen at the meso-scale showing the microstructure. $f$ is volume fraction of the white phase. Right: representative volume element $V$ seen at the macro-scale showing fluctuations in the local volume fraction $f(\boldsymbol{x})$.

To put this information in a more rigorous setting, these composite are considered as three-scale materials exhibiting heterogeneities at different scales hereafter called the micro, meso and macro-scales. These scales are characterized by different lengths $d, a$ and $L$ (see figure 1) corresponding respectively to the size of a single inhomogeneity $(d)$, to the size of clusters of inhomogeneities $(a)$ and to the size of a very large representative volume element $(L)$.

At the smallest scale (micro) the composite is highly heterogeneous, as shown in figure 1a. At the intermediate scale (meso) the sharp micro-inhomogeneities are "smeared out" as shown in figure $1 \mathrm{~b}$ and the composite appears now as an intermediate composite, or meso-composite, comprised of different constituents with a mesostructure representing the different regions, with different volume fraction of constituents, in the actual composite. Finally, at the largest scale all inhomogeneities are smeared out and the composite is seen as an homogeneous medium, the properties of which being, by definition, the macroscopic or effective properties of the composite. 
Consider for instance a two-phase composite composed of identical spherical inclusions distributed in a surrounding matrix in an almost uniform manner. By almost uniform, it is meant that the local volume fraction of inclusions may vary slightly within a large volume element. In other words, the volume fraction of the inclusions is $f_{0}$ at the level of the volume element. But at a smaller (meso) scale it can fluctuate slightly about this mean value and is described by a variable volume fraction $f(\boldsymbol{x})$. In the present study it is assumed that the microstructures of the different regions are of the same type (inclusion/matrix for instance), so that the volume fraction of one phase is the leading parameter in the microstructure description and that the volumefraction fluctuations at the meso-scale are small :

$$
f(\boldsymbol{x})-f_{0} \ll f_{0} .
$$

Obviously a three-scale composite is also a two-scale composite, the two scales being the micro and the macro scale. Therefore the effective properties of the composite can be obtained by an homogenization scheme operating directly between the micro and the macro scales under the sole assumption that $d \ll L$. Depending on the type of microstructure under consideration, examples of such homogenization schemes are given by the Hashin-Shtrikman estimates, the self-consistent scheme or any other appropriate scheme. However, when the three scales are "well separated", which means that the three lengths further satisfy the string of inequalities $d \ll a \ll L$, the effective properties of the composite can be obtained by splitting the homogenization procedure into two successive steps.

The effective properties at the meso-scale (in the different regions) are determined in a first step. It is assumed here that this step of the procedure can been completed and that the effective meso-properties for the different regions are known under the form of an energy function $\widetilde{w}(f, \varepsilon)$ depending on the volume fraction $f$ of phase 1 . For the sake of definiteness examples of functions $\widetilde{w}$ are given in section 2.3 for voided materials.

\subsection{Microscopic, mesoscopic and macroscopic potentials}

The constitutive behavior of each individual constituent $(\alpha)$ is governed at the microscopic scale by a convex potential or strain-energy function $w^{(\alpha)}(\boldsymbol{e})$ in such a way that the infinitesimal strain (or strain-rate) $\boldsymbol{e}$ and the stress $\boldsymbol{s}$ 
at the microscopic scale ${ }^{2}$ are related by

$$
\boldsymbol{s}=\frac{\partial w^{(\alpha)}}{\partial \boldsymbol{e}}(\boldsymbol{e}) \text { in phase }(\alpha)
$$

A volume element $\Omega$, whose size $a$ is large with respect to the size $d$ of the heterogeneities, is subjected to an average deformation $\varepsilon$. As is classical in homogenization theories, the effective energy of the composite at the mesoscale is given by the variational principle :

$$
\widetilde{w}(\varepsilon)=\operatorname{Inf}_{\boldsymbol{u}^{\prime}} \frac{1}{|\Omega|} \int_{\Omega} w\left(\boldsymbol{e}\left(\boldsymbol{u}^{\prime}\right)\right) d \Omega,
$$

where the trial displacement fields $\boldsymbol{u}^{\prime}$ are such that the average over $\Omega$ of the microscopic strain field $\boldsymbol{e}\left(\boldsymbol{u}^{\prime}\right)$ is equal to $\boldsymbol{\varepsilon}$. The effective energy $\widetilde{w}$ is a convex function of $\varepsilon$.

In a second step a macroscopically representative volume element $V$ is chosen, whose size $L$ is much larger that the size $a$ of the meso-representative volume element $\Omega$. The volume fraction $f(\boldsymbol{x})$ of phase 1 depends on the position $\boldsymbol{x}$ at the meso-scale. The average volume fraction at the macroscopic scale is $f_{0}$. The effective energy is given by (as is classical) :

$$
\widetilde{\widetilde{w}}\left(f_{0}, \overline{\boldsymbol{\varepsilon}}\right)=\operatorname{Inf}_{\boldsymbol{u} \in \mathcal{K}(\overline{\boldsymbol{\varepsilon}})}\langle\widetilde{w}(f(\boldsymbol{x}), \boldsymbol{\varepsilon}(\boldsymbol{u}))\rangle,
$$

where $\mathcal{K}(\overline{\boldsymbol{\varepsilon}})=\left\{\boldsymbol{v}=\overline{\boldsymbol{\varepsilon}} \cdot \boldsymbol{x}+\boldsymbol{v}^{*}, \boldsymbol{v}^{*}\right.$ periodic on $\left.\partial V\right\}$. For definiteness, periodicity conditions have been chosen on $\partial V$ but the type of boundary conditions is not important as long as the volume element $V$ is much larger than the typical length of the fluctuations of $f$ in $V$.

In the rest of the paper it will be assumed that the first step of homogenization has been achieved and that $\widetilde{w}(f, \varepsilon)$ is known, either exactly or through an accurate estimate. Our aim is to examine the difference between $\widetilde{w}$ and $\widetilde{w}$ which stems from the deviations of $f(\boldsymbol{x})$ from $f_{0}$.

\subsection{Mesoscopic energy functions for voided materials}

The main theoretical result obtained in this paper in section 3 will be applied to voided materials in section 4 . At the microscopic scale, voids (phase 1)

$\overline{2}$ Different notations, $\boldsymbol{e}, \boldsymbol{\varepsilon}$ and $\overline{\boldsymbol{\varepsilon}}$ are used in the paper to denote the infinitesimal strain at the micro, meso and macro-scale respectively. Similarly $\boldsymbol{s}, \boldsymbol{\sigma}$ and $\overline{\boldsymbol{\sigma}}$ denote the Cauchy stress at the three different scales. The three quantities are related by classical average relations. 
are distributed in a matrix (phase 2) which is a power-law incompressible material characterized by the strain-energy (or dissipation potential when $\boldsymbol{e}$ is interpreted as a strain-rate) :

$$
w(e)=\frac{\sigma_{0} \varepsilon_{0}}{m+1}\left(\frac{e_{e q}}{\varepsilon_{0}}\right)^{m+1} \text { when } \operatorname{tr}(e)=0, \quad+\infty \quad \text { otherwise. }
$$

The particular case $m=1$ corresponds to a linear-elastic material with shear modulus $\mu=\sigma_{0} / 3 \varepsilon_{0}$. As is well-known the effective energy of linear elastic voided materials are bounded from above by the upper Hashin-Shtrikman bound :

$$
\widetilde{w}(f, \varepsilon) \leq \frac{\sigma_{0}(1-f)}{2 \varepsilon_{0}}\left(\frac{4}{f} \varepsilon_{m}^{2}+\frac{1}{1+\frac{2}{3} f} \varepsilon_{e q}^{2}\right) .
$$

In addition the right hand-side of (2.4) is an accurate estimate of the effective energy at the meso-scale when the microstructure of the material is of the composite sphere assemblage type.

The other extreme case $m=0$ corresponds to a rigid-plastic matrix obeying the von Mises criterion with flow stress $\sigma_{0}$. Bounds and estimates for the effective energy of ideally-plastic voided materials have been obtained by means of various variational methods (Ponte Castañeda (1991, 1992), Willis (1991), Suquet $(1992,1993)$ ). However the strain-energy functions obtained by these methods are known to be inaccurate especially at high stress triaxialities (although they are rigorous upper bounds). The most widely used model for porous ideally-plastic materials is due to Gurson (1977) and based on the approximate analysis of a single hollow sphere. The yield function proposed by this author to describe the flow surface of voided ideally-plastic materials reads as :

$$
\widetilde{F}(f, \boldsymbol{\sigma})=\frac{\sigma_{e q}^{2}}{\sigma_{0}}+2 f \cosh \left(\frac{3}{2} \frac{\sigma_{m}}{\sigma_{0}}\right)-1-f^{2} \leq 0 .
$$

The associated energy (or dissipation) function at the meso-scale can be calculated as :

$$
\widetilde{w}(f, \varepsilon)=\operatorname{Sup}_{\boldsymbol{\sigma}, \tilde{F}(\boldsymbol{\sigma}) \leq 0} \boldsymbol{\sigma}: \boldsymbol{\varepsilon} .
$$

Straightforward algebra shows that the energy $\widetilde{w}$ associated with Gurson's criterion (2.5) reads as :

$$
\widetilde{w}(f, \varepsilon)=\sigma_{0} \int_{f}^{1}\left(\frac{4 \varepsilon_{m}^{2}}{y^{2}}+\varepsilon_{e q}^{2}\right)^{1 / 2} \mathrm{~d} y .
$$

Following the work of Gurson (1977), Perrin (1992) proved that (2.6) is an upper bound for the effective potential of a hollow sphere subjected to uniform displacements on its boundary. The potential (2.6) remains a rigorous upper bound for the effective potential of any composite assemblage of hollow spheres with porosity $f$. Numerical studies (see Tvergaard, 1990, for a review) show that this upper bound is also an accurate estimate for spherical voids which are 
periodically distributed in an ideally-plastic matrix. However it is not known if it remains an upper bound for more general microstructures. For instance, it is not known whether the Gurson's potential remains an upper bound, or an accurate estimate, when the voids shapes are not spherical.

For intermediate values of $m$, Leblond et al. (1994) and Gărăjeu et al. (2000), generalizing the analysis of Gurson with the same velocity fields, have proposed a strain-energy function which reads :

$$
\widetilde{w}(f, \varepsilon)=\frac{\sigma_{0}}{(m+1) \varepsilon_{0}^{m}} \int_{f}^{1}\left(\frac{4 \varepsilon_{m}^{2}}{y^{2}}+\varepsilon_{e q}^{2}\right)^{\frac{m+1}{2}} \mathrm{~d} y .
$$

When $m=0$ the strain-energy (2.7) reduces to the Gurson's energy (2.6). When $m=1$ it coincides with the upper Hashin-Shtrikman bound (2.4) for hydrostatic loadings but exceeds slightly this bound for deviatoric loadings. Section 4 will deal with potentials in the form (2.7).

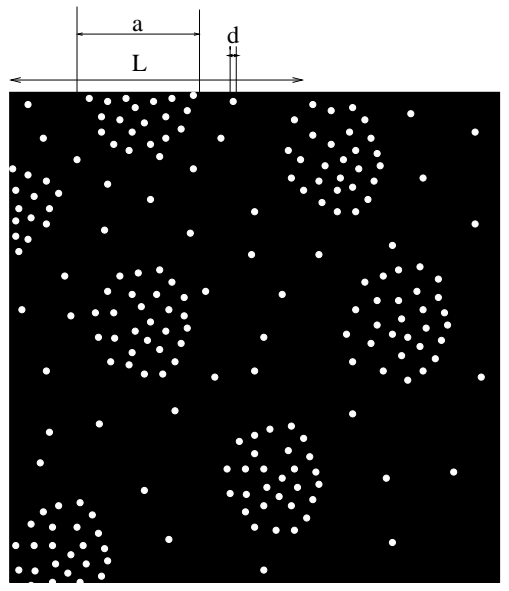

(a)

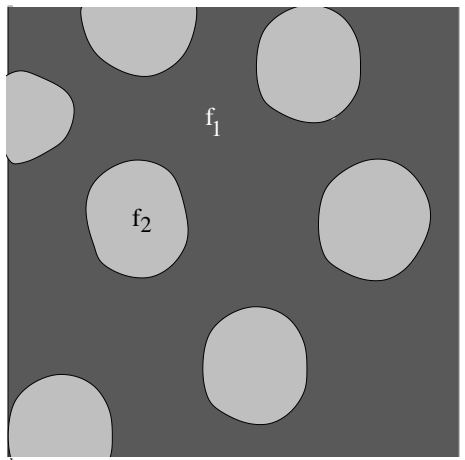

(b)

Fig. 2. Two uniform volume fractions. (a) Actual microstructure, (b) mesostructure.

The question addressed in this study, when specialized to voided materials, can be formulated in the following way. Assume that the microstructure of the voided materials under study can be reasonably considered as a generalized composite sphere assemblage (isolated voids in a matrix). Void clusters can be seen as regions in the r.v.e. where the volume fraction $f_{2}$ of the composite spheres is above the average porosity $f$ and regions where it is below, as schematically depicted in figure 2 a (for clarity the difference in the local volume fractions $f_{1}$ and $f_{2}$ has been much exaggerated in this figure). The corresponding microstructure is not strictly speaking a composite sphere assemblage, but is a generalized composite sphere assemblage in the sense that it results from the piling-up of two different classes of self-similar spheres with two different porosities $f_{1}$ and $f_{2}$. How does the effective potential of such a generalized asemblage differ from that of the corresponding microstructure 
where all the self-similar spheres have the same porosity? A numerical approach to the question would be to consider the mesostructure shown in figure $2 \mathrm{~b}$. Then considering that each region is governed by the Gurson criterion (2.5), one could compute numerically the (macroscopic) effective yield surface of the material. This is not the direction that we have pursued here. Instead we have developed an analytical approach, based on the assumption that the volume fractions $f_{1}$ and $f_{2}$ do not differ too much from $f$. This restriction is counter-balanced by the fact that our analytical results do not depend on the form of the potential and gives qualitative results which shed light on the role, positive or negative, of volume-fraction fluctuations.

\section{A general result for small fluctuations in the local volume frac- tion of constituents}

\subsection{Asymptotic expansion}

As recalled in the introduction, the homogenization procedure for three-scale composites involves a first step by which the mesoscopic effective energy $\widetilde{w}(f, \boldsymbol{\varepsilon})$ is determined. Once the first step of homogenization is completed, in exact or approximate form, it remains to perform the second step of homogenization, i.e. to achieve the meso-macro transition. At the meso-scale the local volume fraction $f$ of phase 1 exhibits fluctuations about its average:

$$
f_{t}(\boldsymbol{x})=f_{0}+t \delta f(\boldsymbol{x}), \quad \text { with } \quad\langle\delta f\rangle=0 .
$$

The parameter $t$ serves to measure the amplitude of the fluctuations about the mean value $f_{0}$. The fluctuations are assumed to be small so that $t$ is a small parameter.

Then, the macroscopic effective energy of the composite, which depends on the small parameter $t$, is defined as

$$
\widetilde{\widetilde{w}}(t, \overline{\boldsymbol{\varepsilon}})=\operatorname{Inf}_{\boldsymbol{u} \in \mathcal{K}(\overline{\boldsymbol{\varepsilon}})}\left\langle\widetilde{w}\left(f_{t}, \boldsymbol{\varepsilon}(\boldsymbol{u})\right)\right\rangle=\left\langle\widetilde{w}\left(f_{t}, \boldsymbol{\varepsilon}\left(\boldsymbol{u}_{t}\right)\right)\right\rangle
$$

where $\mathcal{K}(\overline{\boldsymbol{\varepsilon}})=\left\{\boldsymbol{v}=\overline{\boldsymbol{\varepsilon}} \cdot \boldsymbol{x}+\boldsymbol{v}^{*}, \boldsymbol{v}^{*}\right.$ periodic $\}$. The fields $\boldsymbol{u}_{t}$ and $\boldsymbol{\varepsilon}\left(\boldsymbol{u}_{t}\right)$ are the local displacement and associated strain fields at the meso-scale induced by appropriate boundary conditions generating an average strain $\bar{\varepsilon}$ in $V$. It will be assumed that $\widetilde{\widetilde{w}}(., t)$ and $\boldsymbol{u}_{t}$ are continuously differentiable functions of $t$. Since $t$ is small, it is appropriate to look for a perturbation series expansion of $\widetilde{\widetilde{w}}$ about $t=0$. 
The main result result of this section is the following expansion

$\widetilde{\widetilde{w}}(t, \bar{\varepsilon})=\widetilde{w}\left(f_{0}, \bar{\varepsilon}\right)+\frac{t^{2}}{2}\left(\left\langle\delta f^{2}\right\rangle \frac{\partial^{2} \widetilde{w}}{\partial f^{2}}\left(f_{0}, \bar{\varepsilon}\right)-\frac{\partial^{2} \widetilde{w}}{\partial f \partial \boldsymbol{\varepsilon}}(\bar{\varepsilon}): \mathbb{H}: \frac{\partial^{2} \widetilde{w}}{\partial f \partial \boldsymbol{\varepsilon}}(\bar{\varepsilon})\right)+O\left(t^{3}\right)$,

where $\mathbb{H}$ is a fourth-order tensor specified by (3.12).

\section{Comments:}

1. In general, fluctuations in the volume fraction have no first-order effect on the effective potential but have a second-order effect. This is true as long as the Taylor expansion (3.3) is legitimate. In particular, when the tensor $\mathbb{H}$ becomes singular, the expansion (3.3) is no more valid.

2. Fluctuations have a first-order effect on the local fields, reflected by the first-order terms $\dot{\boldsymbol{u}}_{0}$ and $\dot{\boldsymbol{\sigma}}_{0}$ (solutions of the problem (3.10).

3. The fluctuations enter this second-order expansion through their covariance $\left\langle\delta f^{2}\right\rangle$ and through the fourth-order tensor $\mathbb{H}$ which depends on the geometrical arrangement of the domains where the perturbations around the mean value $f_{0}$ take place.

4. Fluctuations in volume fraction can have, at second-order, a beneficial or deleterious effect on the effective potential $\widetilde{\widetilde{w}}$, depending on the sign of the second-order term.

5. It should be kept in mind for further consideration that the Taylor expansion(3.3) is valid for small values of the volume fraction fluctuation $(t)$ and can break down if the second-order term blows up (this will the case for rigid-plastic materials under specific loading conditions as studied in detail in section 5. In the same spirit, it should be noted that, although $\widetilde{w}$ and $\widetilde{\widetilde{w}}$ are convex functions of $\varepsilon$ and $\bar{\varepsilon}$ respectively, nothing guarantees that the Taylor expansion truncated at order 2 as given by (3.3), is a convex function with respect to $\bar{\varepsilon}$ when $t$ is not small enough.

Derivation of (3.3): The expansion of $\widetilde{\widetilde{w}}$ in powers of $t$ is formally given by its Taylor expansion at $t=0$. The first derivatives in this expansion may be computed by successive derivation of (3.2); this procedure will naturally involve derivatives of $\boldsymbol{u}_{t}$. The problem to be solved for $\boldsymbol{u}_{t}$ is given by

$$
\boldsymbol{\sigma}_{t}=\frac{\partial \widetilde{w}}{\partial \boldsymbol{\varepsilon}}\left(f_{t}, \boldsymbol{\varepsilon}\left(\boldsymbol{u}_{t}\right)\right), \quad \operatorname{div}\left(\boldsymbol{\sigma}_{t}\right)=\mathbf{0}, \quad \boldsymbol{u}_{t} \in \mathcal{K}(\overline{\boldsymbol{\varepsilon}})
$$

By differentiation of (3.4), it is seen that $\dot{\boldsymbol{u}}_{t}$ (the dot denoting the derivative 
with respect to $t$ ) is, for all $t$, the solution of the systems of equations:

$$
\dot{\boldsymbol{\sigma}}_{t}=\mathbb{L}_{t}: \varepsilon\left(\dot{\boldsymbol{u}}_{t}\right)+\boldsymbol{\tau}_{t}, \quad \operatorname{div}\left(\dot{\boldsymbol{\sigma}}_{t}\right)=\mathbf{0}, \quad \dot{\boldsymbol{u}}_{t} \in \mathcal{K}(\mathbf{0})
$$

where

$$
\mathbb{L}_{t}=\frac{\partial^{2} \widetilde{w}}{\partial \varepsilon \partial \varepsilon}\left(f_{t}, \boldsymbol{\varepsilon}\left(\boldsymbol{u}_{t}\right)\right), \quad \boldsymbol{\tau}_{t}=\delta f(\boldsymbol{x}) \frac{\partial^{2} \widetilde{w}}{\partial f \partial \boldsymbol{\varepsilon}}\left(f_{t}, \boldsymbol{\varepsilon}\left(\boldsymbol{u}_{t}\right)\right) .
$$

$\mathbb{L}_{t}$ is the tensor of instantaneous or tangent moduli. It follows from (3.2) and from Hill's lemma that

$$
\begin{aligned}
\frac{\partial \widetilde{\widetilde{w}}}{\partial t}(t, \overline{\boldsymbol{\varepsilon}}) & =\left\langle\frac{\partial \widetilde{w}}{\partial \boldsymbol{\varepsilon}}\left(f_{t}, \boldsymbol{\varepsilon}\left(\boldsymbol{u}_{t}\right)\right): \boldsymbol{\varepsilon}\left(\dot{\boldsymbol{u}}_{t}\right)\right\rangle+\left\langle\delta f(\boldsymbol{x}) \frac{\partial \widetilde{w}}{\partial f}\left(f_{t}, \boldsymbol{\varepsilon}\left(\boldsymbol{u}_{t}\right)\right)\right\rangle \\
& =\left\langle\delta f(\boldsymbol{x}) \frac{\partial \widetilde{w}}{\partial f}\left(f_{t}, \boldsymbol{\varepsilon}\left(\boldsymbol{u}_{t}\right)\right)\right\rangle .
\end{aligned}
$$

Differentiating again yields:

$$
\frac{\partial^{2} \widetilde{w}}{\partial t^{2}}(t, \overline{\boldsymbol{\varepsilon}})=\left\langle(\delta f(\boldsymbol{x}))^{2} \frac{\partial^{2} \widetilde{w}}{\partial f^{2}}\left(f_{t}, \boldsymbol{\varepsilon}\left(\boldsymbol{u}_{t}\right)\right)\right\rangle+\left\langle\delta f(\boldsymbol{x}) \frac{\partial^{2} \widetilde{w}}{\partial f \partial \boldsymbol{\varepsilon}}\left(f_{t}, \boldsymbol{\varepsilon}\left(\boldsymbol{u}_{t}\right)\right): \boldsymbol{\varepsilon}\left(\dot{\boldsymbol{u}}_{t}\right)\right\rangle .
$$

By means of the system of equations (3.5) satisfied by $\dot{\boldsymbol{u}}_{t}$, one obtains finally that :

$$
\frac{\partial^{2} \widetilde{\widetilde{w}}}{\partial t^{2}}(t, \overline{\boldsymbol{\varepsilon}})=\left\langle(\delta f(\boldsymbol{x}))^{2} \frac{\partial^{2} \widetilde{w}}{\partial f^{2}}\left(f_{t}, \boldsymbol{\varepsilon}\left(\boldsymbol{u}_{t}\right)\right)\right\rangle-\left\langle\varepsilon\left(\dot{\boldsymbol{u}}_{t}\right): \mathbb{L}_{t}: \boldsymbol{\varepsilon}\left(\dot{\boldsymbol{u}}_{t}\right)\right\rangle .
$$

The relations (3.6) and (3.8), which hold for arbitrary values of $t$, give the first two derivatives of $\widetilde{w}$ at $t=0$. The material is homogeneous at the meso-scale at $t=0$. Therefore $\boldsymbol{u}_{0}=\bar{\varepsilon} \cdot \boldsymbol{x}$ and :

$$
\left.\begin{array}{c}
\widetilde{\widetilde{w}}(0, \overline{\boldsymbol{\varepsilon}})=w\left(f_{0}, \overline{\boldsymbol{\varepsilon}}\right), \quad \frac{\partial \widetilde{w}}{\partial t}(0, \overline{\boldsymbol{\varepsilon}})=\langle\delta f\rangle \frac{\partial \widetilde{w}}{\partial f}\left(f_{0}, \overline{\boldsymbol{\varepsilon}}\right)=0 \quad(\text { since }\langle\delta f\rangle=0), \\
\frac{\partial^{2} \widetilde{\widetilde{w}}}{\partial t^{2}}(0, \overline{\boldsymbol{\varepsilon}})=\left\langle(\delta f(\boldsymbol{x}))^{2}\right\rangle \frac{\partial^{2} \widetilde{w}}{\partial f^{2}}\left(f_{0}, \overline{\boldsymbol{\varepsilon}}\right)-\left\langle\boldsymbol{\varepsilon}\left(\dot{\boldsymbol{u}}_{0}\right): \mathbb{L}: \boldsymbol{\varepsilon}\left(\dot{\boldsymbol{u}}_{0}\right)\right\rangle, \\
\text { where } \quad \mathbb{L}=\frac{\partial^{2} \widetilde{w}}{\partial \boldsymbol{\varepsilon} \partial \boldsymbol{\varepsilon}}\left(f_{0}, \overline{\boldsymbol{\varepsilon}}\right) .
\end{array}\right\}
$$

In these relations, $\dot{\boldsymbol{u}}_{0}$ is the solution of the linear thermoelasticity problem:

$$
\left.\begin{array}{r}
\dot{\boldsymbol{\sigma}}_{0}=\mathbb{L}: \boldsymbol{\varepsilon}\left(\dot{\boldsymbol{u}}_{0}\right)+\boldsymbol{\tau}, \quad \operatorname{div}\left(\dot{\boldsymbol{\sigma}}_{0}\right)=\mathbf{0}, \quad \dot{\boldsymbol{u}}_{0} \in \mathcal{K}(\mathbf{0}), \\
\text { where } \boldsymbol{\tau}(\boldsymbol{x})=\delta f(\boldsymbol{x}) \frac{\partial^{2} \widetilde{w}}{\partial f \partial \boldsymbol{\varepsilon}}\left(f_{0}, \overline{\boldsymbol{\varepsilon}}\right) .
\end{array}\right\}
$$

Given that the modulus tensor $\mathbb{L}$ is constant, problem (3.10) is a standard linear thermoelasticity problem for a homogeneous material with a distribution 
of body forces determined by the polarization field $\boldsymbol{\tau}$. Its solution can be expressed as $\boldsymbol{\varepsilon}\left(\dot{\boldsymbol{u}}_{0}\right)=-\mathbb{\Gamma} * \boldsymbol{\tau}$, where $\mathbb{\Gamma}$ is the Green operator associated with $\mathbb{L}$.

When the fluctuations are piecewise uniform, i.e. uniform in $N$ "regions" $V_{r}$ with characteristic function $\chi^{(r)}$, the polarization field is piecewise constant with :

$$
\boldsymbol{\tau}(\boldsymbol{x})=\sum_{r=1}^{N} \chi^{(r)}(\boldsymbol{x}) \boldsymbol{\tau}^{(r)}, \quad \boldsymbol{\tau}^{(r)}=\frac{\partial^{2} \widetilde{w}}{\partial f \partial \boldsymbol{\varepsilon}}\left(f_{0}, \overline{\boldsymbol{\varepsilon}}\right) \delta f^{(r)} .
$$

Then, taking advantage of Hill's lemma, one obtains that :

$$
\left\langle\varepsilon\left(\dot{\boldsymbol{u}}_{0}\right): \mathbb{L}: \boldsymbol{\varepsilon}\left(\dot{\boldsymbol{u}}_{0}\right)\right\rangle=-\left\langle\boldsymbol{\tau}: \boldsymbol{\varepsilon}\left(\dot{\boldsymbol{u}}_{0}\right)\right\rangle=\langle\boldsymbol{\tau}: \mathbb{\Gamma} * \boldsymbol{\tau}\rangle=\sum_{r=1}^{N} \sum_{s=1}^{N} \boldsymbol{\tau}^{(r)}: \mathbb{\Gamma}^{(r s)}: \boldsymbol{\tau}^{(s)},
$$

where

$$
\mathbb{\Gamma}^{(r s)}=\left\langle\chi^{(r)} \mathbb{\Gamma} * \chi^{(s)}\right\rangle .
$$

In conclusion, when fluctuations have zero average around the mean value $f_{0}$, the expansion (3.3) is obtained with :

$$
\mathbb{H}=\sum_{r=1}^{N} \sum_{s=1}^{N} \delta f^{(r)}: \mathbb{\Gamma}^{(r s)}: \delta f^{(s)}
$$

\subsection{Inclusion-rich zones and inclusion-poor zones}

Assume that the fluctuations are piecewise uniform on only two subdomains $(N=2)$ (see figure 2), whose distribution is compatible with the overall isotropy of the composite. It follows from the fact that the fluctuations around the mean value $f_{0}$ have zero average that $\delta f^{(1)}$ and $\delta f^{(2)}$ should have the following form :

$$
\delta f^{(1)}=\frac{\Delta f}{c^{(1)}}, \quad \delta f^{(2)}=-\frac{\Delta f}{c^{(2)}},
$$

where $c^{(1)}$ and $c^{(2)}$ denote the volume fractions of the domains where the perturbations take place. Then

$$
\delta f(x)=\frac{\Delta f}{c^{(1)} c^{(2)}}\left(\chi_{1}(\boldsymbol{x})-c^{(1)}\right) \quad \text { and } \quad\left\langle\delta f^{2}\right\rangle=\frac{(\Delta f)^{2}}{c^{(1)} c^{(2)}} .
$$

The tensor $\mathbb{H}$ is computed from (3.12) with $\delta f^{(1)}$ and $\delta f^{(2)}$ given above and, since in the case of a two-phases composite the microstructural tensors $\mathbb{\Gamma}^{(r s)}$ have particular properties ::

$$
\mathbb{\Gamma}^{(12)}=\mathbb{\Gamma}^{(21)}=-\mathbb{\Gamma}^{(11)}=-\mathbb{\Gamma}^{(22)},
$$


it follows :

$$
\mathbb{H}=\frac{(\Delta f)^{2}}{c^{(1)} c^{(2)}} \mathbb{P}, \quad \text { with } \quad \mathbb{P}=\frac{1}{c^{(1)} c^{(2)}}\left\langle\chi^{(1)} \mathbb{\Gamma} * \chi^{(1)}\right\rangle
$$

and therefore the second order derivative of the effective potential is :

$$
\frac{\partial^{2} \widetilde{\widetilde{w}}}{\partial t^{2}}(\bar{\varepsilon}, 0)=\frac{(\Delta f)^{2}}{c^{(1)} c^{(2)}}\left(\frac{\partial^{2} \widetilde{w}}{\partial f^{2}}\left(f_{0}, \bar{\varepsilon}\right)-\frac{\partial^{2} \widetilde{w}}{\partial f \partial \boldsymbol{\varepsilon}}(\overline{\boldsymbol{\varepsilon}}): \mathbb{P}: \frac{\partial^{2} \widetilde{w}}{\partial f \partial \boldsymbol{\varepsilon}}(\overline{\boldsymbol{\varepsilon}})\right)
$$

The two domains on which the perturbation take place can be chosen arbitrarily to a certain extent (with the constraint that their distribution should be such that the composite is macroscopically isotropic). The influence of this choice on the effective potential $\widetilde{w}$ is "measured" by the product $c^{(1)} c^{(2)}$ and the largest effect is obtained when $c^{(1)}=c^{(2)}=1 / 2$.

\subsection{Materials with mesoscopic isotropy}

Assume that the composites under consideration are isotropic at the mesoscopic scale. Their mesoscopic energy $\widetilde{w}$ depends only on the first three invariants of $\varepsilon$. Assume for further simplicity that $\widetilde{w}$ depends only on the first two invariants :

$$
\widetilde{w}(f, \varepsilon)=\widetilde{w}\left(f, \varepsilon_{m}, \varepsilon_{e q}\right) .
$$

If, additionally, the geometrical distribution of the two fluctuation domains is statistically uniform and isotropic (i.e. the two-point correlation functions depend only on the distance between any two points in the composite), then following Willis (1981), the tensor $\mathbb{P}$ defined in (3.13) is related to the instantaneous elastic tensor $\mathbb{L}(3.9)$ by :

$$
\mathbb{P}=\left.\frac{1}{4 \pi} \int_{\| \boldsymbol{\xi}_{\|=1}} \boldsymbol{\xi} \otimes \mathbf{N}(\boldsymbol{\xi}) \otimes \boldsymbol{\xi}\right|_{s y m} d s(\boldsymbol{\xi})
$$

with $\mathbf{N}(\boldsymbol{\xi})=(\mathbf{K}(\boldsymbol{\xi}))^{-1}$ is the inverse of the acoustic tensor $\mathbf{K}(\boldsymbol{\xi})=\boldsymbol{\xi}$.LL. $\boldsymbol{\xi}$.

The tensor $\mathbb{L}$ is the second derivative of the potential $\widetilde{w}(\varepsilon)$ with respect to $\varepsilon$ evaluated at $\bar{\varepsilon}$. The first derivative reads ${ }^{3}$ :

$$
\frac{\partial \widetilde{w}}{\partial \boldsymbol{\varepsilon}}(\overline{\boldsymbol{\varepsilon}})=\frac{1}{3} \frac{\partial \widetilde{w}}{\partial \varepsilon_{m}}(\overline{\boldsymbol{\varepsilon}}) \boldsymbol{i}+\frac{2}{3} \frac{\partial \widetilde{w}}{\partial \varepsilon_{e q}}(\overline{\boldsymbol{\varepsilon}}) \frac{\overline{\boldsymbol{e}}}{\varepsilon_{e q}}
$$

$\overline{3}$ For simplicity we shall drop, in what follows, the dependence on $f$ which is just a parameter. 
where $\boldsymbol{i}$ is the second-order tensor identity and $\overline{\boldsymbol{e}}$ is the deviator of $\overline{\boldsymbol{\varepsilon}}$. The second derivative reads

$$
\frac{\partial^{2} \widetilde{w}}{\partial \varepsilon^{2}}(\overline{\boldsymbol{\varepsilon}})=\frac{1}{3} \frac{\partial^{2} \widetilde{w}}{\partial \varepsilon_{m}^{2}}(\overline{\boldsymbol{\varepsilon}}) \mathbb{J}+\frac{2}{3} \frac{\partial^{2} \widetilde{w}}{\partial \varepsilon_{e q}^{2}}(\overline{\boldsymbol{\varepsilon}}) \mathbb{E}+\frac{2}{3} \frac{1}{\varepsilon_{e q}} \frac{\partial \widetilde{w}}{\partial \varepsilon_{e q}}(\overline{\boldsymbol{\varepsilon}}) \mathbb{F}+\frac{2}{9} \frac{\partial^{2} \widetilde{w}}{\partial \varepsilon_{m} \partial \varepsilon_{e q}}(\overline{\boldsymbol{\varepsilon}}) \mathbb{G}
$$

where

$$
\mathbb{J}=\frac{1}{3} \boldsymbol{i} \otimes \boldsymbol{i}, \quad \mathbb{E}=\frac{2}{3} \widehat{\boldsymbol{e}} \otimes \widehat{\boldsymbol{e}}, \quad \mathbb{F}=\mathbb{I}-\mathbb{J}-\mathbb{E}, \quad \mathbb{G}=\boldsymbol{i} \otimes \widehat{\boldsymbol{e}}+\widehat{\boldsymbol{e}} \otimes \boldsymbol{i},
$$

with $\widehat{\boldsymbol{e}}=\frac{\overline{\boldsymbol{e}}}{\bar{\varepsilon}_{e q}}$. In the above relations $\mathbb{I}$ is the fourth-order tensor representing the identity between symmetric fourth-order tensors, $\mathbb{J}$ is the projector over spherical tensors and therefore $\mathbb{K}=\mathbb{I}-\mathbb{J}$ is the projector over deviatoric tensors, $\mathbb{E}$ is the projector over $\widehat{\boldsymbol{e}}$ and $\mathbb{E}+\mathbb{F}=\mathbb{K}$. e materials. The fourthorder tensors $\mathbb{E}$ and $\mathbb{F}$ were introduced by Ponte Castañeda (1996) to express the tangent moduli in incompressible nonlinear materials. Another tensor $\mathbb{G}$ is necessary here to account for compressibility effects. Set

$$
k=\frac{1}{9} \frac{\partial^{2} \widetilde{w}}{\partial \varepsilon_{m}^{2}}(\bar{\varepsilon}), \quad \lambda=\frac{1}{3} \frac{\partial^{2} \widetilde{w}}{\partial \varepsilon_{e q}^{2}}(\bar{\varepsilon}), \quad \mu=\frac{1}{3} \frac{1}{\bar{\varepsilon}_{e q}} \frac{\partial \widetilde{w}}{\partial \varepsilon_{e q}}(\bar{\varepsilon}), \quad \gamma=\frac{1}{9} \frac{\partial^{2} \widetilde{w}}{\partial \varepsilon_{m} \partial \varepsilon_{e q}}(\bar{\varepsilon}) .
$$

Then

$$
\mathbb{L}=3 k \mathbb{J}+2 \lambda \mathbb{E}+2 \mu \mathbb{F}+2 \gamma \mathbb{G}
$$

Let $\boldsymbol{\eta}=\widehat{\boldsymbol{e}} . \boldsymbol{\xi}$. Since

$$
\begin{gathered}
\boldsymbol{\xi} . \mathbb{J} . \boldsymbol{\xi}=\frac{1}{3} \boldsymbol{\xi} \otimes \boldsymbol{\xi}, \quad \boldsymbol{\xi} . \mathbb{E} . \boldsymbol{\xi}=\frac{2}{3} \boldsymbol{\eta} \otimes \boldsymbol{\eta}, \\
\boldsymbol{\xi} . \mathbb{F} . \boldsymbol{\xi}=\frac{1}{2}\left(\boldsymbol{i}+\frac{1}{3} \boldsymbol{\xi} \otimes \boldsymbol{\xi}-\frac{4}{3} \boldsymbol{\eta} \otimes \boldsymbol{\eta}\right), \quad \boldsymbol{\xi} . \mathbb{G} . \boldsymbol{\xi}=\boldsymbol{\eta} \otimes \boldsymbol{\xi}+\boldsymbol{\xi} \otimes \boldsymbol{\eta},
\end{gathered}
$$

the acoustic tensor $\mathbf{K}(\boldsymbol{\xi})$ reads as :

$\mathbf{K}(\boldsymbol{\xi})=\boldsymbol{\xi} . \mathbb{L} . \boldsymbol{\xi}=\mu\left[\boldsymbol{i}+\left(\frac{k}{\mu}+\frac{1}{3}\right) \boldsymbol{\xi} \otimes \boldsymbol{\xi}+\frac{2 \gamma}{\mu}(\boldsymbol{\eta} \otimes \boldsymbol{\xi}+\boldsymbol{\xi} \otimes \boldsymbol{\eta})+\frac{4}{3}\left(\frac{\lambda}{\mu}-1\right) \boldsymbol{\eta} \otimes \boldsymbol{\eta}\right]$

and can be put in the form :

$$
\mathbf{K}=\boldsymbol{i}+\lambda_{1} \boldsymbol{\xi} \otimes \boldsymbol{\xi}+\lambda_{2}(\boldsymbol{\xi} \otimes \boldsymbol{\eta}+\boldsymbol{\eta} \otimes \boldsymbol{\xi})+\lambda_{3} \boldsymbol{\eta} \otimes \boldsymbol{\eta},
$$

with

$$
\lambda_{1}=\frac{k}{\mu}+\frac{1}{3}, \quad \lambda_{2}=\frac{2 \gamma}{\mu}, \quad \lambda_{3}=\frac{4}{3}\left(\frac{\lambda}{\mu}-1\right) .
$$

According to lemma 8 of appendix A the inverse of $\boldsymbol{K}$ can be expressed as :

$$
\mathbf{N}(\boldsymbol{\xi})=\frac{1}{\mu}\left\{\boldsymbol{i}-\frac{1}{\Delta}\left[\Lambda_{1} \boldsymbol{\xi} \otimes \boldsymbol{\xi}+\Lambda_{2}(\boldsymbol{\xi} \otimes \boldsymbol{\eta}+\boldsymbol{\eta} \otimes \boldsymbol{\xi})+\Lambda_{3} \boldsymbol{\eta} \otimes \boldsymbol{\eta}\right]\right\},
$$


where the detailed expressions of $\Delta$ and of the $\Lambda_{i}$ 's are given in appendix A. The polarization field $\boldsymbol{\tau}$ specializes to

$\boldsymbol{\tau}=\frac{\partial^{2} \widetilde{w}}{\partial f \partial \boldsymbol{\varepsilon}}\left(f_{0}, \overline{\boldsymbol{\varepsilon}}\right)=\tau_{m} \boldsymbol{i}+\frac{2}{3} \tau_{e q} \widehat{\boldsymbol{e}}, \quad \tau_{m}=\frac{1}{3} \frac{\partial^{2} \widetilde{w}}{\partial f \partial \varepsilon_{m}}\left(f_{0}, \overline{\boldsymbol{\varepsilon}}\right), \quad \tau_{e q}=\frac{\partial^{2} \widetilde{w}}{\partial f \partial \varepsilon_{e q}}\left(f_{0}, \overline{\boldsymbol{\varepsilon}}\right)$.

To determine the second-order expansion (3.3) of the effective potential is sufficient to evaluate $\boldsymbol{\tau}: \mathbb{P}: \boldsymbol{\tau}$ in (3.14) rather than computing $\mathbb{P}$ itself :

$$
\begin{aligned}
\boldsymbol{\tau}: \mathbb{P}: \boldsymbol{\tau} & =\frac{1}{4 \pi} \int_{\| \boldsymbol{\xi}_{\|=1}} \boldsymbol{\tau}:(\boldsymbol{\xi} \otimes \mathbf{N}(\boldsymbol{\xi}) \otimes \boldsymbol{\xi}): \boldsymbol{\tau} \mathrm{d} s(\boldsymbol{\xi}) \\
& =\frac{1}{4 \pi} \int_{\| \boldsymbol{\xi}_{\|=1}}(\boldsymbol{\tau} \cdot \boldsymbol{\xi}) \cdot \mathbf{N}(\boldsymbol{\xi}) \cdot(\boldsymbol{\tau} \cdot \boldsymbol{\xi}) \mathrm{d} s(\boldsymbol{\xi})
\end{aligned}
$$

Note that $\boldsymbol{\tau} . \boldsymbol{\xi}=\tau_{m} \boldsymbol{\xi}+\frac{2}{3} \tau_{e q} \boldsymbol{\eta}$ and therefore the integrand reads

$$
(\boldsymbol{\tau} \cdot \boldsymbol{\xi}) \cdot \mathbf{N}(\boldsymbol{\xi}) .(\boldsymbol{\tau} \cdot \boldsymbol{\xi})=\tau_{m}^{2} \boldsymbol{\xi} \cdot \mathbf{N}(\boldsymbol{\xi}) \cdot \boldsymbol{\xi}+\frac{4}{3} \tau_{m} \tau_{e q} \boldsymbol{\xi} \cdot \mathbf{N}(\boldsymbol{\xi}) \cdot \boldsymbol{\eta}+\frac{4}{9} \tau_{e q}^{2} \boldsymbol{\eta} \cdot \mathbf{N}(\boldsymbol{\xi}) \cdot \boldsymbol{\eta} .
$$

Straightforward algebra gives :

$$
\begin{aligned}
& \boldsymbol{\xi} . \mathbf{N}(\boldsymbol{\xi}) \cdot \boldsymbol{\xi}=\frac{1}{\mu}\left(1-\frac{\Lambda_{1}+2 \Lambda_{2} p+\Lambda_{3} p^{2}}{\Delta}\right)=\frac{1}{\mu \Delta}\left[1+\lambda_{3}\left(b^{2}-p^{2}\right)\right] \\
& \boldsymbol{\xi} \cdot \mathbf{N}(\boldsymbol{\xi}) \cdot \boldsymbol{\eta}=\frac{1}{\mu}\left[p-\frac{\Lambda_{1} p+\Lambda_{2}\left(b^{2}+p^{2}\right)+\Lambda_{3} p b^{2}}{\Delta}\right]=\frac{1}{\mu \Delta}\left[p-\lambda_{2}\left(b^{2}-p^{2}\right)\right], \\
& \boldsymbol{\eta} \cdot \mathbf{N}(\boldsymbol{\xi}) \cdot \boldsymbol{\eta}=\frac{1}{\mu}\left(b^{2}-\frac{\Lambda_{1} p^{2}+2 \Lambda_{2} p b^{2}+\Lambda_{3} b^{4}}{\Delta}\right)=\frac{1}{\mu \Delta}\left[b^{2}+\lambda_{1}\left(b^{2}-p^{2}\right)\right] .
\end{aligned}
$$

with the notations of appendix A $(b=\|\boldsymbol{\eta}\|, p=\boldsymbol{\xi} \cdot \boldsymbol{\eta})$. Finally the integral reads

$\boldsymbol{\tau}: \mathbb{P}: \boldsymbol{\tau}=\frac{1}{4 \pi} \int_{\|\boldsymbol{\xi}\|=1} \frac{\tau_{m}^{2}+\frac{4}{3} \tau_{m} \tau_{e q} p+\frac{4}{9} \tau_{e q}^{2} b^{2}+\left(\tau_{m}^{2} \lambda_{3}-\frac{4}{3} \tau_{m} \tau_{e q} \lambda_{2}+\frac{4}{9} \tau_{e q}^{2} \lambda_{1}\right)\left(b^{2}-p^{2}\right)}{\mu \Delta} \mathrm{d} s(\boldsymbol{\xi})$

This integral cannot be computed explicitly in general. As remarked in other circumstances by Ponte Castañeda and Suquet (1998), it depends strongly on the third invariant of the strain tensor $\bar{\varepsilon}$. Indeed, $\bar{\varepsilon}$ appears in the integral (3.21) through $p=\boldsymbol{\xi} . \hat{\boldsymbol{e}} . \boldsymbol{\xi}$ and $b=(\boldsymbol{\xi} . \hat{\boldsymbol{e}} \cdot \hat{\boldsymbol{e}} \cdot \boldsymbol{\xi})^{1 / 2}$ which are independent of the reference frame. Relative to its principal axes, $\widehat{\boldsymbol{e}}$ may be represented (Kachanov, 1971) in terms of a single parameter $\omega, 0 \leqslant \omega<\pi / 3$, the principal values of $\widehat{\boldsymbol{e}}$ taking the form

$$
\widehat{e}_{1}=-\cos \left(\omega-\frac{\pi}{3}\right), \quad \widehat{e}_{2}=-\cos \left(\omega+\frac{\pi}{3}\right), \quad \widehat{e}_{3}=\cos \omega .
$$


The angle $\omega$ is related to the third invariant of the macroscopic strain $\bar{\varepsilon}$. Indeed, the determinant of $\widehat{\boldsymbol{e}}$, which is closely related to this third invariant, can be expressed in terms of $\omega$ as $\operatorname{det}(\widehat{\boldsymbol{e}})=\cos (3 \omega) / 4$. The values $\omega=0$ and $\omega=\pi / 6$ correspond to an axisymmetric strain (deviatoric part of a uniaxial deformation) and simple shear, respectively.

\section{Comments:}

Our results show that the perturbed potential $\widetilde{\widetilde{w}}$ depends on the third invariant of the strain even though the unperturbed potential $\widetilde{w}(3.15)$ has no such dependence. This raises the question of the validity of assumption (3.15). The influence of the third invariant of stress (or strain) on the effective properties of porous materials has been the subject of contradictory observations. On the one hand Duva and Hutchinson (1984) and Richelsen and Tvergaard (1994) have observed a rather weak effect of this invariant. On the other hand theoretical results (for weakly contrasted materials) by Ponte Castañeda and Suquet (1998) have shown potentially strong effects. Since this is still an open question (to the best of the authors' knowledge) and by lack of an accurate model including third invariant effects, the assumption (3.15) was made.

\section{Nonlinear porous materials}

\subsection{The main result}

This section is devoted to the influence of isotropic porosity fluctuations on the effective behaviour of nonlinear porous materials. Our main observation is the following one:

Conjecture 1: For any porous material whose effective behaviour is described by the Gurson-type potential (2.6) or (2.7), the second-order perturbation due to fluctuations in porosity is always negative.

In other words, fluctuations in porosity always weaken porous materials with effective properties described by (2.7).

We have a complete analytical proof of conjecture 1 in three different cases : first when $m=1$, second for arbitrary $m$ when the macroscopic strain is either a pure dilatation or a simple shear (see section 4.3), and third when $m=0$ and when the macroscopic strain triaxiality $T=\bar{\varepsilon}_{m} / \bar{\varepsilon}_{e q}$ is larger than 1 . In the remaining cases, numerical simulations presented in section 4.5 strongly support the conjecture's validity. 
The perturbation of the effective potential $\widetilde{\widetilde{w}}(\bar{\varepsilon}, t)$ induced by porosity fluctuations can be measured by the normalized quantity:

$$
\delta(f, \overline{\boldsymbol{\varepsilon}})=\left(\frac{\partial^{2} \widetilde{w}}{\partial f^{2}}(f, \overline{\boldsymbol{\varepsilon}})-\frac{\partial^{2} \widetilde{w}}{\partial f \partial \boldsymbol{\varepsilon}}(f, \overline{\boldsymbol{\varepsilon}}): \mathbb{P}: \frac{\partial^{2} \widetilde{w}}{\partial f \partial \boldsymbol{\varepsilon}}(f, \overline{\boldsymbol{\varepsilon}})\right) / \widetilde{w}(f, \overline{\boldsymbol{\varepsilon}})
$$

This quantity does not depend on the amplitude of the fluctuations $t \Delta f$ but depends, through the tensor $\mathbb{P}$, on the geometrical arrangement of the domains where the fluctuations take place. Conjecture 1 can be alternatively formulated by saying that $\delta$ is negative for all $m$ 's and all macroscopic strains $\bar{\varepsilon}$.

It should be noted that since $\widetilde{w}$ is a positively homogeneous function of $\varepsilon$ of degree $m+1, \mathbb{L}$ and $\mathbb{P}$ are positively homogeneous functions of $\bar{\varepsilon}$ with degree $m-1$ and $1-m$ respectively. It follows that $\delta$ depends on the macroscopic strain $\bar{\varepsilon}$ only through the strain triaxiality-ratio defined as

$$
T=\frac{\bar{\varepsilon}_{m}}{\bar{\varepsilon}_{e q}}
$$

and through the angle $\omega$ defined by (3.22) and related to the third invariant of the strain. Therefore $\delta=\delta(f, T, \omega)$.

\subsection{Linear materials}

In the linear case $m=1$, the expression of $\delta$ is quite simple. The potential $\widetilde{w}(f, \varepsilon)$ takes the form :

$$
\widetilde{w}(f, \boldsymbol{\varepsilon})=\frac{\sigma_{0}}{2 \varepsilon_{0}}(1-f)\left(\frac{4 \varepsilon_{m}^{2}}{f}+\varepsilon_{e q}^{2}\right)
$$

The relations (3.17) give $\gamma=0$ and $\lambda=\mu$ and therefore the elastic tensor $\mathbb{L}$ (3.18) and the corresponding $\mathbb{P}$ tensor (3.16) whose expression can be found in Willis (1981) are isotropic :

$$
\mathbb{L}=\frac{2}{3} \frac{\sigma_{0}}{\varepsilon_{0}}(1-f)\left[\frac{2}{f} \mathbb{J}+\mathbb{K}\right], \quad \mathbb{P}=\frac{1}{\frac{2}{3} \frac{\sigma_{0}}{\varepsilon_{0}}\left(1-f^{2}\right)}\left[\frac{f}{2} \mathbb{J}+\frac{2+3 f}{5} \mathbb{K}\right],
$$

where $\mathbb{K}$ is the projector over deviatoric tensors (to avoid confusion, the second order acoustic tensor is always denoted in the paper by $\mathbf{K}(\boldsymbol{\xi})$ ). The polarization field $\boldsymbol{\tau}$ and the term $\boldsymbol{\tau}: \mathbb{P}: \boldsymbol{\tau}$ reduce to

$$
\boldsymbol{\tau}=-\frac{2}{3} \frac{\sigma_{0}}{\varepsilon_{0}}\left(\frac{2 \bar{\varepsilon}_{m}}{f^{2}} \boldsymbol{i}+\bar{\varepsilon}_{e q} \widehat{\boldsymbol{e}}\right), \quad \boldsymbol{\tau}: \mathbb{P}: \boldsymbol{\tau}=\frac{\sigma_{0}}{\varepsilon_{0}} \frac{1}{1-f^{2}}\left(\frac{4 \bar{\varepsilon}_{m}^{2}}{f^{3}}+\frac{2+3 f}{5} \bar{\varepsilon}_{e q}^{2}\right) .
$$


Finally, the second-order perturbation $\delta$ defined in (4.1) reads :

$$
\delta=\frac{\sigma_{0}}{\varepsilon_{0}} \frac{1}{1-f^{2}}\left(-\frac{4 \bar{\varepsilon}_{m}^{2}}{f}-\frac{2+3 f}{5} \bar{\varepsilon}_{e q}^{2}\right) / \widetilde{w}(f, \bar{\varepsilon}) \leq 0 .
$$

Conjecture 1 is therefore proved for linear materials.

Motivated by this result and by the numerical simulations presented in section 4.5 , a second conjecture can be formulated :

Conjecture 2: For any porous materials whose effective behaviour is described by the Gurson-type potential (2.7), the second-order perturbation $\delta$ is an increasing function of $m$ when $0 \leq m \leq 1 \quad(\delta(0) \leq \delta(m) \leq \delta(1))$.

Conjecture 1 is a consequence of conjecture 2 and of the result established in the linear case since, if conjecture 2 holds true, one has for fixed porosity $f$ and fixed applied strain $\bar{\varepsilon}$ :

$$
\delta(m) \leq \delta(1)<0
$$

\subsection{Proof of conjectures 1 and 2 for specific loading conditions}

The evaluation of $\delta$ for specific loading conditions requires detailed expressions for the different terms in (4.1) which are given in appendix B.

\subsubsection{Pure dilatation}

When the macroscopic strain is a pure dilatation $\left(\bar{\varepsilon}_{e q}=0, T=\infty\right)$, the potential $\widetilde{w}(f, \bar{\varepsilon})$ reduces to (appendix B.1):

$$
\widetilde{w}(f, \bar{\varepsilon})=\frac{\sigma_{0}}{\varepsilon_{0}^{m}}\left(\frac{2\left|\bar{\varepsilon}_{m}\right|}{f}\right)^{m+1} \frac{f\left(1-f^{m}\right)}{m(m+1)}
$$

and its second derivative with respect to $f$ reads:

$$
\frac{\partial^{2} \widetilde{w}}{\partial f^{2}}(f, \bar{\varepsilon})=\frac{\sigma_{0}}{\varepsilon_{0}^{m}} \frac{1}{f}\left(\frac{2\left|\bar{\varepsilon}_{m}\right|}{f}\right)^{m+1}
$$

Finally, using results of the appendix $\mathrm{B}, \delta_{\infty}=\delta_{\mid T=\infty}$ can be expressed in closed form :

$$
\delta_{\infty}(f, m)=\frac{m+1}{f^{2}} \frac{m}{1-f^{m}} \frac{(m-1) f^{m}-f^{2}}{1-f^{2}+(1-m)\left(1-f^{m}\right)}
$$


$\delta_{\infty}$ depends on $f$ and $m$ only. As the product of three increasing functions

$$
m \rightarrow \frac{m+1}{f^{2}}, \quad m \rightarrow \frac{m}{1-f^{m}}, \quad m \rightarrow \frac{(m-1) f^{m}-f^{2}}{1-f^{2}+(1-m)\left(1-f^{m}\right)},
$$

the first two being positive, $\delta_{\infty}$ is an increasing function of $m$.

Conjecture 2 and consequently conjecture 1 are therefore proved for purely dilatational deformations.

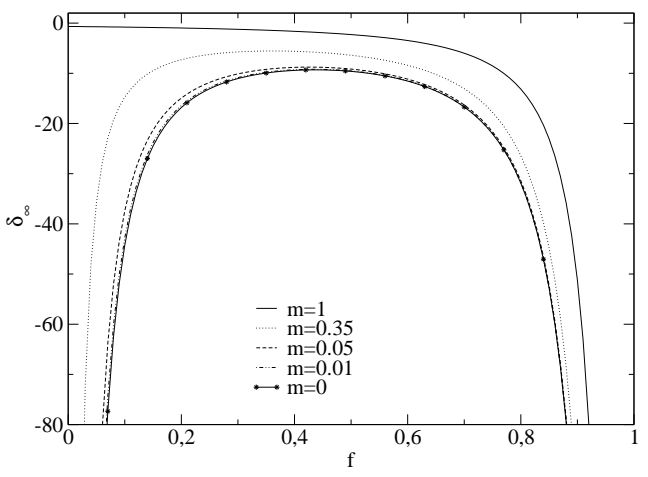

Fig. 3. Normalized second-order perturbation $\delta_{\infty}$ as a function of $f$ for different values of $m$

The normalized second-order perturbation $\delta_{\infty}$ is plotted in Figure 3 as a function of $f$ for different values of $m$. For completeness, the figure shows $\delta_{\infty}$ when $f$ ranges from 0 to 1 . It it is therefore implicitely assumed that Gurson's model remains accurate in this range of volume fraction $f$. In other words, it is assumed that the porosity remains closed even for large porosities. If this is not the case, and in particular if the porosity becomes open over a certain percolation threshold, the asymptotic expansion (3.3) remains valid upon use of a proper expression for $\widetilde{w}$ accounting for this change in microstructure.

The singularity observed in these curves in the vicinity of $f=0$ is due to the fact that the material becomes incompressible in the limit as $f$ goes to 0 . The potential $\widetilde{w}$, its second derivative with respect to $f$ and the different terms entering the expression of the second-order perturbation become infinite in this limit. Similarly, when $f$ approaches 1 the potential $\widetilde{w}$ tends to 0 (the material loses its carrying capacity) and the normalization by $\widetilde{w}(f, \bar{\varepsilon})$ creates a singularity for $\delta_{\infty}$. 


\subsubsection{Simple shear}

When the overall deformation is a simple shear $\left(\bar{\varepsilon}_{m}=0\right.$, i.e. $\left.T=0\right)$ the potential $\widetilde{w}$ is an affine function of $f$,

$$
\widetilde{w}(f, \bar{\varepsilon})=\frac{\sigma_{0} \bar{\varepsilon}_{e q}^{m+1}}{(m+1) \varepsilon_{0}^{m}}(1-f)
$$

and therefore $\frac{\partial^{2} \widetilde{w}}{\partial f^{2}}(f, \bar{\varepsilon})=0$.

Then, using appendix B.2 one obtains the expression of the perturbation $\delta_{0}=$ $\delta_{\mid T=0}$ :

$$
\delta_{0}=-\frac{1}{3 \pi} \frac{m+1}{(1-f)^{2}} \int_{\|\boldsymbol{\xi}\|=1} \frac{\|\widehat{\mathbf{e}} \cdot \boldsymbol{\xi}\|^{2}-\frac{4+f}{4(1+f)}(\boldsymbol{\xi} . \widehat{\mathbf{e}} \cdot \boldsymbol{\xi})^{2}}{1+\frac{4}{3}(m-1)\left(\|\widehat{\mathbf{e}} \cdot \boldsymbol{\xi}\|^{2}-\frac{4+f}{4(1+f)}(\boldsymbol{\xi} . \widehat{\mathbf{e}} \cdot \boldsymbol{\xi})^{2}\right)} \mathrm{d} s(\boldsymbol{\xi})
$$

which is a function of $f$ and $\omega$. Note that for all values of $f$ and $\omega, \delta_{0}$ is an increasing function on $m$. Indeed, $m \rightarrow \frac{m+1}{(1-f)^{2}}$ is an increasing and positive function and, since

$$
\|\widehat{\mathbf{e}} . \boldsymbol{\xi}\|^{2}-\frac{4+f}{4(1+f)}(\boldsymbol{\xi} . \widehat{\mathbf{e}} . \boldsymbol{\xi})^{2} \geq\|\widehat{\mathbf{e}} \cdot \boldsymbol{\xi}\|^{2}-(\boldsymbol{\xi} . \widehat{\mathbf{e}} \cdot \boldsymbol{\xi})^{2} \geq 0
$$

the function

$$
m \rightarrow-\frac{1}{3 \pi} \int_{\|\boldsymbol{\xi}\|=1} \frac{\|\widehat{\mathbf{e}} \cdot \boldsymbol{\xi}\|^{2}-\frac{4+f}{4(1+f)}(\boldsymbol{\xi} . \hat{\mathbf{e}} \cdot \boldsymbol{\xi})^{2}}{1+\frac{4}{3}(m-1)\left(\|\hat{\mathbf{e}} \cdot \boldsymbol{\xi}\|^{2}-\frac{4+f}{4(1+f)}(\boldsymbol{\xi} . \widehat{\mathbf{e}} \cdot \boldsymbol{\xi})^{2}\right)}
$$

is an increasing function.

Conjecture 2 and consequently conjecture 1 are therefore proved for simple shear.

The normalized second-order perturbation $\delta_{0}$ for $\omega=0$ is plotted in Figure 4a for different values of $m$. Again, the large values of $\delta$ observed when $f$ approaches 1 are due to the fact that the normalization factor $\widetilde{w}(f, \bar{\varepsilon})$ tends to 0 in this limit. The plots in Figure $4 \mathrm{~b}$ show $\delta_{0}$ as a function of $f$ when $m=0$ and for different values of $\omega$, except for $\omega=\frac{\pi}{6}$ where the integral in (4.4) is divergent for any $f$ (as proved in section 5.1).

Compared to the order of magnitude of $\delta_{\infty}$ shown in figure 3 , the second order perturbation $\delta_{0}$ is small for most $f$ 's and $\omega$ 's which means that the Gurson potential is almost insensitive to small perturbations in the void volume fraction when the overall strain is a pure shear. 


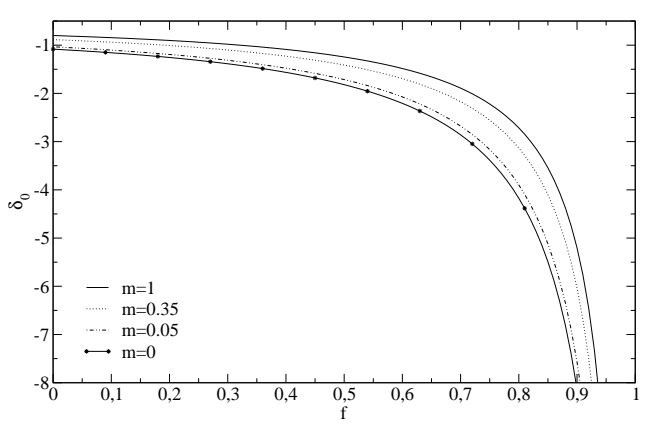

(a)

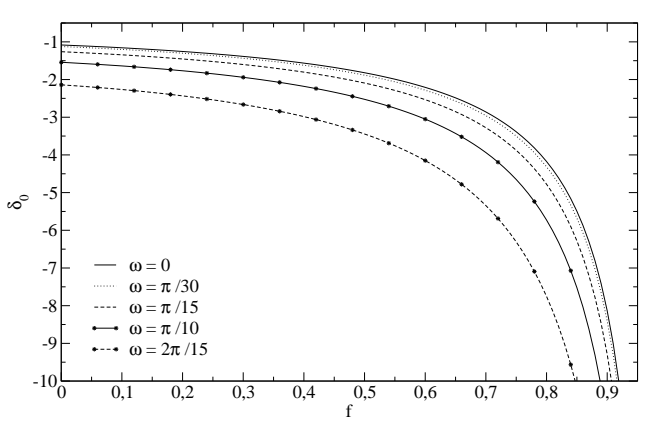

(b)

Fig. 4. Normalized second-order perturbation $\delta_{0}$ : (a) when $\omega=0$, as a function of $f$ and different values of $m$; (b) when $m=0$, as a function of $f$ and different values of $\omega$

4.4 Proof of conjecture 1 for rigid-plastic materials and $|T| \geq 1$.

In this section we prove that $\delta$ is negative when $m=0$ and $|T| \geq 1$.

The proof requires in particular an estimate of (3.21). Note first that both the numerator and the denominator of the integrand in (3.21) are positive since, according to section 5.1 the acoustic tensor $\mathbf{K}(\boldsymbol{\xi})$ and its inverse $\mathbf{N}(\boldsymbol{\xi})$ are positive definite when $|T| \geq 1 / 2$. The idea of the proof is to find a uniform upper bound $\Delta^{\max }$ for $\Delta(\boldsymbol{\xi})$ which is independent of $\boldsymbol{\xi}$. Such an inequality for $\Delta$ will lead to a lower bound for $\boldsymbol{\tau}: \mathbb{P}: \tau$ :

$$
\begin{aligned}
& \boldsymbol{\tau}: \mathbb{P}: \boldsymbol{\tau} \geq \\
& \frac{1}{4 \pi} \frac{1}{\mu \Delta^{\max }} \int_{\|\boldsymbol{\xi}\|=1} \tau_{m}^{2}+\frac{4}{3} \tau_{m} \tau_{e q} p+\frac{4}{9} \tau_{e q}^{2} b^{2}+\left(\tau_{m}^{2} \lambda_{3}-\frac{4}{3} \tau_{m} \tau_{e q} \lambda_{2}+\frac{4}{9} \tau_{e q}^{2} \lambda_{1}\right)\left(b^{2}-p^{2}\right) \mathrm{d} s(\boldsymbol{\xi}) .
\end{aligned}
$$

The integral on the right-hand-side of the inequality can be computed in closed form :

$$
\boldsymbol{\tau}: \mathbb{P}: \boldsymbol{\tau} \geq \frac{1}{\mu \Delta^{\max }}\left[\frac{4}{9}\left(\frac{1}{2}+\frac{3}{10} \lambda_{1}\right) \tau_{e q}^{2}-\frac{2}{5} \lambda_{2} \tau_{e q} \tau_{m}+\left(1+\frac{3}{10} \lambda_{3}\right) \tau_{m}^{2}\right] .
$$

Then using (B.4) and (B.2) with $m=0$ (note that in the case $m=0, a(T, f)$ simplifies to $\left.a(T, f)=\sqrt{4 T^{2}+f^{2}}\right)$ the above inequality reads

$$
\boldsymbol{\tau}: \mathbb{P}: \boldsymbol{\tau} \geq \frac{1}{\Delta^{\max }} \frac{4 \sigma_{0} \varepsilon_{e q}}{15 f^{2}(a(T, 1)-a(T, f))}\left[3+2 \frac{a(T, f)}{a(T, 1)}+3 \frac{f^{2}\left(f^{2}-1\right)}{a(T, f)^{2}}\right]
$$

The normalized second perturbation $\delta$ has the same sign as $\frac{\partial^{2} \widetilde{w}}{\partial f^{2}}(f, \overline{\boldsymbol{\varepsilon}})-\boldsymbol{\tau}$ : $\mathbb{P}: \tau$. We get from the above inequality :

$$
\frac{\partial^{2} w}{\partial f^{2}}(f, \overline{\boldsymbol{\varepsilon}})-\boldsymbol{\tau}: \mathbb{P}: \boldsymbol{\tau} \leq \frac{4 \sigma_{0} \varepsilon_{e q}}{f^{2} a(T, f)}\left\{T^{2}-\frac{1}{\Delta^{\max }} \frac{B}{5(1-B)}\left[1+\frac{2}{3} B+f^{2}\left(1-\frac{1}{B^{2}}\right)\right]\right\}
$$


where $B=a(T, f) / a(T, 1)$ satisfies the identity $\left(f^{2}-1\right) / a(T, f)^{2}=1-1 / B^{2}$. It remains to specify the upper bound $\Delta^{\max }$ in this inequality. For this purpose the following technical lemma is proved in Appendix C :

Lemma 1 When $|T| \geq \frac{1}{2}$ then $\Delta \leq \Delta^{\max }=\frac{4}{3 a(T, 1) a(T, f)}(1+2|T|)^{2}$.

Then, upon substitution of $\Delta^{\max }$ in the above inequality, we obtain that :

$$
\frac{\partial^{2} \widetilde{w}}{\partial f^{2}}(f, \overline{\boldsymbol{\varepsilon}})-\boldsymbol{\tau}: \mathbb{P}: \boldsymbol{\tau} \leq \frac{4 \sigma_{0} \varepsilon_{e q}}{f^{2} a(T, f)(1+2|T|)^{2}} \mathcal{D}
$$

where :

$$
\mathcal{D}=T^{2}(1+2|T|)^{2}-\frac{3 a(T, f)^{2}}{20(1-B)}\left[1+\frac{2}{3} B+f^{2}\left(1-\frac{1}{B^{2}}\right)\right] .
$$

It remains to prove that $\mathcal{D}$ is negative. Another technical result is used :

Lemma $2 \mathcal{D}$ is a decreasing function of $f$.

Lemma 2 is proved in the Appendix D.

According to this lemma the maximum of $\mathcal{D}$ is reached for $f=0$. Note that for $f=0, a(T, 0)=2|T|, B=2|T| / a(T, 1)$ and $\frac{1}{1-B}=a(T, 1)(a(T, 1)+2|T|)$. It follows that:

$$
\begin{aligned}
\mathcal{D} & \leq T^{2}(1+2|T|)^{2}-\frac{3 T^{2}\left(1+\frac{2}{3} B\right)}{5(1-B)} \\
& =T^{2}\left[(1+2|T|)^{2}-a(T, 1)(a(T, 1)+2|T|) \frac{3+2 B}{5}\right] \\
& =\frac{2 T^{2}}{5}\left(1+10|T|-5|T| \sqrt{1+4 T^{2}}\right) \\
(\text { for }|T| \geq 1) & \leq \frac{2 T^{2}}{5}(1+10|T|-5 \sqrt{5}|T|) \leq(11-5 \sqrt{5}) \frac{2 T^{2}}{5}<0
\end{aligned}
$$

This completes the proof of the inequality $\delta \leq 0$ for $m=0$ and $|T| \geq 1$.

\subsection{Numerical evaluation of the second-order perturbation}

The above sections prove conjectures 1 and 2 in a certain range of macroscopic strains $\bar{\varepsilon}$ and of rate-sensitivity exponents $m$. To investigate the remaining cases the normalized second-order perturbation $\delta$ is computed numerically for porous materials governed by a Gurson-type potential using the relations (3.21) and (B.4). 


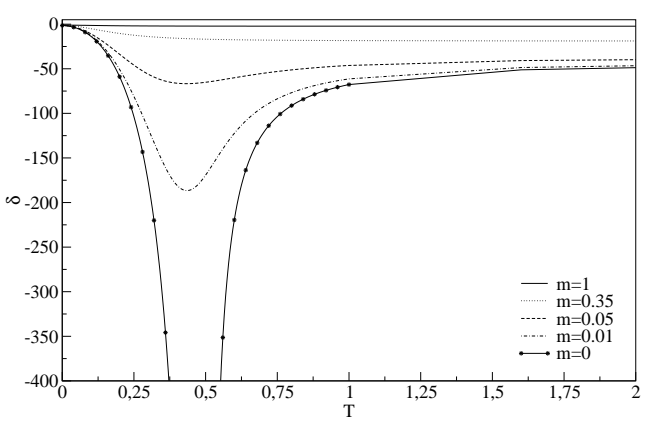

a)

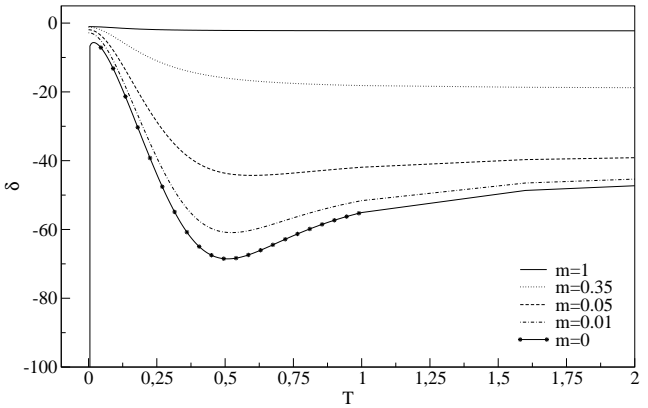

b)

Fig. 5. Second-order perturbation $\delta$ for $f_{0}=0.1$, as a function of $T$ for different values of $m$ : (a) $\omega=0$; (b) $\omega=\pi / 6$

The variations of $\delta$ as a function of the strain rate triaxiality $T$ are shown in Figure 5 for different values of the exponent $m$. The average porosity is $f_{0}=0.1$. Figure 5a corresponds to uniaxial tension $(\omega=0)$, whereas Figure 5 b corresponds to simple slip $(\omega=\pi / 6)$. The following points are worth noticing :

- First it is observed that $\delta$ is always negative. This supports conjecture 1. It is also noted that $\delta$ is an increasing function of $m$. This observation supports conjecture 2 .

- Second, when $m=0$ the plots indicate that $\delta$ becomes singular for specific values of the strain triaxiality-ratio depending on $\omega$. When $\omega=0$ the singularity is observed for $T=1 / 2$ and when $\omega=\pi / 6$, the singularity is observed for $T=0$.

- Another aspect of the singularity of $\delta$ in the rigid-plastic case is reported in figure 6 where $\delta$ is plotted as a function of $\omega$ for a given strain triaxiality $T$ ( $m=0$ for all plots). When the macroscopic strain triaxiality $T$ is less than $1 / 2$ (figure $6 \mathrm{a}$ ), there is a specific value of $\omega$ for which $\delta$ becomes singular. In contrast, when $T$ is larger than $1 / 2$ no singularity of $\omega$ is observed.

Most of these observations will be confirmed by analytical results in section 5 .

\section{Singularity of the second-order perturbation for rigid-plastic ma- terials}

It was observed in the numerical investigations reported in section 4.5 that the second-order term in the expansion (3.3) can be singular for rigid-plastic materials. When $\widetilde{w}$ is a twice-differentiable function of $f$ (which is usually the case), this singularity can only come from the $\mathbb{P}$ tensor in (3.14) and therefore from a degeneracy of the acoustic tensor $\mathbf{K}(\boldsymbol{\xi})=\boldsymbol{\xi}$.LL $(\boldsymbol{\varepsilon})$. $\boldsymbol{\xi}$. The acoustic tensor 


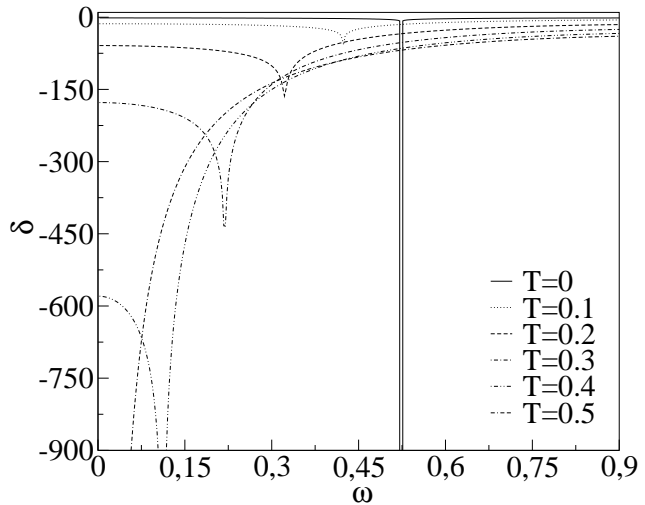

(a)

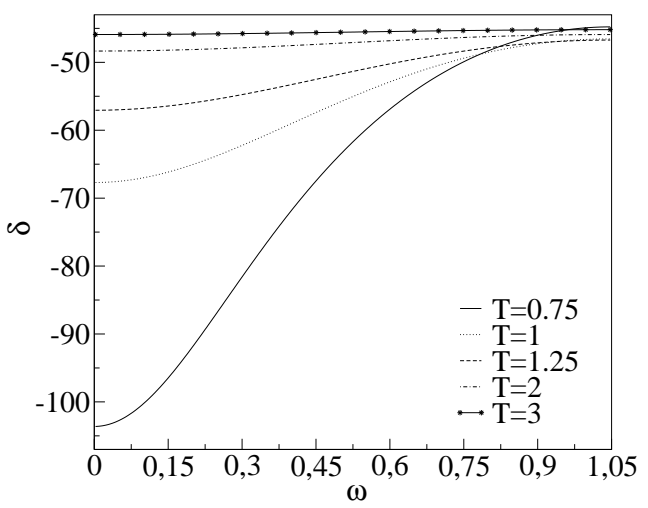

(b)

Fig. 6. Second-order perturbation $\delta$ for $f_{0}=0.1$, and $m=0$ as a function of $\omega$ for different values of $T$ : (a) $0 \leq T \leq 0.5$; (b) $0.5<T<10$

is always positive (by convexity of $\widetilde{w}$ ) but can become degenerate when its determinant vanishes. This possibility is investigated here both for rigid-plastic and viscous materials. Considering an isotropic convex energy $\widetilde{w}$ in the form (3.15), we prove the following results :

- When $m=0$, the acoustic tensor $\mathbf{K}(\boldsymbol{\xi})$ can only be singular when $\varepsilon$ is a rank-one tensor.

- When $m>0$, the acoustic tensor $\mathbf{K}(\boldsymbol{\xi})$ is always positive definite.

In conclusion, the second-order perturbation $\delta$ can only be singular for rigidplastic materials $(m=0)$ and only for rank-one macroscopic deformations.

\subsection{Rigid-plastic materials $m=0$}

The energy function $\widetilde{w}$ is positively homogeneous of degree $m+1$ and therefore satisfies :

$$
\widetilde{w}(\lambda \varepsilon)=\lambda^{m+1} \widetilde{w}(\varepsilon) \text { for all positive } \lambda^{\prime} \mathrm{s} .
$$

Differentiating (5.5) twice with respect to $\lambda$ and taking the resulting relation for $\lambda=1$ gives :

$$
\varepsilon: \mathbb{L}(\varepsilon): \varepsilon=m(m+1) \widetilde{w}(\varepsilon),
$$

where $\mathbb{L}(\varepsilon)$ is the tensor of tangent moduli (second derivative of $\widetilde{w}$ ) defined by (3.9). It results from the convexity of $\widetilde{w}$ that the fourth-order symmetric tensor $\mathbb{L}(\varepsilon)$ is positive (in the sense of quadratic forms). But when $m=0$ the relation (5.6) shows that $\mathbb{L}(\varepsilon)$ is not positive definite, since (5.6) reduces in this case to :

$$
\varepsilon: \mathbb{L}(\varepsilon): \varepsilon=0, \text { for all second-order symmetric } \varepsilon .
$$

The tangent moduli $\mathbb{L}$ being singular, the associated acoustic tensor is likely to be singular as well, leading to $\Delta=\operatorname{det}(\mathbf{K}(\boldsymbol{\xi}))=0$ and explaining the 
singularity of $\delta$ observed in plots 6 .

This intuitive guess is in fact not always true, but is true for specific strains as rigorously shown by the main result of this section.

Theorem 1 Let $w$ be an isotropic energy function in the form (3.15) which is convex and positively homogeneous of degree 1 . Let $\mathbb{L}(\varepsilon)$ be its tensor of tangent moduli for a given strain $\boldsymbol{\varepsilon}$ and let $\mathbf{K}(\boldsymbol{\xi})$ be the acoustic tensor associated with $\mathbb{L}(\boldsymbol{\varepsilon})$ through the relation $\mathbf{K}(\boldsymbol{\xi})=\boldsymbol{\xi} \cdot \mathbb{L}(\boldsymbol{\varepsilon}) \cdot \boldsymbol{\xi}$. Then, there exists a unit vector $\boldsymbol{\xi}$ such that $\operatorname{det}(\mathbf{K}(\boldsymbol{\xi}))=0$ if and only if $\boldsymbol{\varepsilon}$ is a rank-one tensor.

Recall that $\varepsilon$ is a rank-one symmetric second-order tensor if there exist two vectors $\mathbf{a}$ and $\mathbf{b}$ in $\mathbb{R}^{3}$ such that $\varepsilon=\mathbf{a} \otimes_{s} \mathbf{b}$.

A more thorough characterization of symmetric rank-one tensors is needed before proceeding to the proof of theorem 1.

Lemma 3 Let $\varepsilon$ be an element of $\mathbb{R}_{s}^{3 \times 3}$, space of symmetric second-order tensors, with eigenvalues $\varepsilon_{1} \leq \varepsilon_{2} \leq \varepsilon_{3}$. Then

a) $\varepsilon$ is rank-one if and only if $\varepsilon_{2}=0$.

b) Let $T=\frac{\varepsilon_{m}}{\varepsilon_{e q}}$ be the triaxiality-ratio of $\varepsilon$. Then, for all rank-one tensors $\varepsilon$, $|T| \leq \frac{1}{2}$.

c) Conversely, for every $|T| \leq \frac{1}{2}$, there exists a unique (up to a rotation and a multiplicative factor) rank-one tensor whose triaxiality-ratio is $T$.

Proof ${ }^{4}$ of a): First we prove that for all rank-one tensors $\varepsilon$, the intermediate eigenvalue $\varepsilon_{2}$ is equal to 0 , from what it follows that the two other eigenvalues are of opposite sign.

Let $\boldsymbol{\varepsilon}=\mathbf{a} \otimes_{s} \mathbf{b}$. Whenever $\mathbf{a}$ and $\mathbf{b}$ are parallel, two of the eigenvalues of $\boldsymbol{\varepsilon}$ are equal to 0 and the assertion is proved. When $\mathbf{a}$ and $\mathbf{b}$ Result 5.3are not collinear, a basis of $\mathbb{R}^{3}$ can be formed by considering the three vectors:

$$
\mathbf{e}_{1}=\frac{\mathbf{a}}{\|\mathbf{a}\|}, \quad \mathbf{e}_{2}=\frac{\mathbf{a} \wedge \mathbf{b}}{\|\mathbf{a} \wedge \mathbf{b}\|}, \quad \mathbf{e}_{3}=\mathbf{e}_{1} \wedge \mathbf{e}_{2}
$$

In this basis, $\mathbf{a}, \mathbf{b}$ and $\varepsilon$ write respectively

$$
\mathbf{a}=\left[\begin{array}{c}
\|\mathbf{a}\| \\
0 \\
0
\end{array}\right], \quad \mathbf{b}=\left[\begin{array}{c}
b_{1} \\
0 \\
b_{3}
\end{array}\right], \quad[\boldsymbol{\varepsilon}]=\left[\begin{array}{ccc}
\|\mathbf{a}\| b_{1} & 0 & \frac{1}{2}\|\mathbf{a}\| b_{3} \\
0 & 0 & 0 \\
\frac{1}{2}\|\mathbf{a}\| b_{3} & 0 & 0
\end{array}\right] .
$$

$\overline{4}$ An alternative proof of this result can be found in Bhattacharya (2003, Result $5.3)$. 
The eigenvalues of $\boldsymbol{\varepsilon}$ solve the characteristic equation :

$$
\lambda\left[\lambda\left(\lambda-\|\mathbf{a}\| b_{1}\right)-\frac{\|\mathbf{a}\|^{2} b_{3}^{2}}{4}\right]=0
$$

It follows from this expression that one of the eigenvalues of $\varepsilon$ is 0 (the associated eigenvector is $\mathbf{e}_{2}$ ), and that the other two eigenvalues are of opposite sign since their product is negative $\left(-\frac{\|\mathbf{a}\|^{2} b_{3}^{2}}{4} \leq 0\right)$. Therefore $\varepsilon_{1} \leq 0, \varepsilon_{2}=0$ and $\varepsilon_{3} \geq 0$.

Conversely, assume that the eigenvalues of $\varepsilon$ satisfy the string of inequalities $\varepsilon_{1} \leq \varepsilon_{2}=0 \leq \varepsilon_{3}$. A direct calculation shows that $\varepsilon=\mathbf{a} \otimes_{s} \mathbf{b}$ where the components of $\mathbf{a}$ and $\mathbf{b}$ in the principal basis of $\boldsymbol{\varepsilon}$ are:

$$
\mathbf{a}=\frac{1}{\sqrt{\varepsilon_{3}-\varepsilon_{1}}}\left[\begin{array}{c}
\sqrt{-\varepsilon_{1}} \\
0 \\
\sqrt{\varepsilon_{3}}
\end{array}\right], \quad \mathbf{b}=\sqrt{\varepsilon_{3}-\varepsilon_{1}}\left[\begin{array}{c}
-\sqrt{-\varepsilon_{1}} \\
0 \\
\sqrt{\varepsilon_{3}}
\end{array}\right] .
$$

This completes the proof of point a).

Proof of b): Let $\varepsilon$ be a rank-one tensor (such that $\varepsilon_{1} \leq \varepsilon_{2}=0 \leq \varepsilon_{3}$ according to part a) of the lemma). Its first two invariants and its triaxiality-ratio read as :

$$
\varepsilon_{m}=\frac{\varepsilon_{1}+\varepsilon_{3}}{3}, \quad \varepsilon_{e q}=\frac{2}{3} \sqrt{\varepsilon_{1}^{2}+\varepsilon_{3}^{2}-\varepsilon_{1} \varepsilon_{3}}, \quad T=\frac{1+x}{2 \sqrt{1+x^{2}-x}}, \quad x=\frac{\varepsilon_{1}}{\varepsilon_{3}} \leq 0 .
$$

The function $x \rightarrow T(x)$ is a strictly increasing function on ] $-\infty, 0]$ since its derivative $T^{\prime}=\frac{3-3 x}{2\left(1+x^{2}-x\right)^{3 / 2}}$ is strictly positive on this interval. Therefore the minimum and maximum of $T$ are attained at the boundary of the interval, when $x=0$ and $x \rightarrow-\infty$ :

$$
\lim _{x \rightarrow \infty} T=-\frac{1}{2} \leq T(x) \leq T(0)=\frac{1}{2} .
$$

This completes the proof of point b).

Proof of c): Note that for given $|T| \leq \frac{1}{2}$ and unknown $x$, the equation (5.8) has a unique solution $x_{T}$ on the interval $\left.]-\infty, 0\right]$ because of the strict monotonicity of $T(x)$ on this interval. Choose $\varepsilon_{2}=0$ and pick-up arbitrarily $\varepsilon_{3}>0$ (if $T=-\frac{1}{2}$ then $x_{T} \rightarrow-\infty, \varepsilon_{3}=0$ and $\varepsilon_{1}<0$ is chosen arbitrarily). Then the problem is reduced to finding a rank-one tensor $\varepsilon$ with eigenvalues $\left(\varepsilon_{1}=\right.$ $\left.\varepsilon_{3} x_{T}, 0, \varepsilon_{3}\right)$. According to point a) such a rank-one tensor exists.

Corollary 1 There exists a unique axisymmetric rank-one tensor (up to a 
rotation and a multiplicative factor):

$$
\varepsilon=\varepsilon \boldsymbol{n} \otimes \boldsymbol{n},
$$

where $\boldsymbol{n}$ is an arbitrary unit vector and $\varepsilon$ an arbitrary scalar.

Indeed, let $\varepsilon$ be an axisymmetric second-order rank-one tensor and let $\varepsilon_{1}=\varepsilon_{2}$ and $\varepsilon_{3}$ denote its eigenvalues. Then, either $\varepsilon_{1} \leq \varepsilon_{3}$ or $\varepsilon_{1} \geq \varepsilon_{3}$. In both cases, since $\varepsilon$ is rank one, it follows, from the lemma 3 a), that $\varepsilon_{1}=\varepsilon_{2}=0$. Therefore two eigenvalues of $\varepsilon$ vanish and the corollary is proved.

Note that the triaxiality-ratio $T$ of this tensor is $|T|=\frac{1}{2}$.

Lemma 4 Let $w$ and $\mathbb{L}(\varepsilon)$ be as in Theorem 1 and such that the coefficients $k, \lambda, \mu$ and $\gamma$ defined by (3.17) satisfy $\mu \neq 0$ and $k, \gamma$ and $\lambda$ are not all equal to 0 .

Then the set $\mathcal{L}_{\text {min }}=\left\{\boldsymbol{\theta} \in \mathbb{R}_{s}^{3 \times 3}\right.$ such that $\left.\boldsymbol{\theta}: \mathbb{L}: \boldsymbol{\theta}=0\right\}\left(\mathcal{L}_{\text {min }}\right.$ is the set where the quadratic form defined by $\mathbb{L}$ attains its minimum) is a one-dimensional space spanned by $\varepsilon$.

Proof of lemma 4: We first show that $\mathcal{L}_{\text {min }}$ coincides with the kernel of $\mathbb{L}$ :

$$
\mathcal{L}_{\text {min }}=\operatorname{Ker}(\mathbb{L})=\{\boldsymbol{\theta} \mid \mathbb{L}: \boldsymbol{\theta}=0\} .
$$

The inclusion $\operatorname{Ker}(\mathbb{L}) \subset \mathcal{L}_{\text {min }}$ is straightforward. Conversely, let $\boldsymbol{\theta}$ be such that $\boldsymbol{\theta}: \mathbb{L}: \boldsymbol{\theta}=0$. Then $\boldsymbol{\theta}$ is a minimum point for the function $\boldsymbol{\theta} \rightarrow \frac{1}{2} \boldsymbol{\theta}: \mathbb{L}: \boldsymbol{\theta}$ since $\mathbb{L}$ is positive (from the convexity of $w$ ). As such it satisfies the stationarity condition $\frac{\partial}{\partial \boldsymbol{\theta}}\left(\frac{1}{2} \boldsymbol{\theta}: \mathbb{L}: \boldsymbol{\theta}\right)=0$. Therefore $\mathbb{L}: \boldsymbol{\theta}=0$.

The next step is to prove that if $\boldsymbol{\theta}$ is such that $\mathbb{L}: \boldsymbol{\theta}=0$, its deviator is parallel to $\widehat{\boldsymbol{e}}$. Using the standard decomposition, $\boldsymbol{\theta}=\theta_{m} \boldsymbol{i}+\boldsymbol{\theta}^{d}$, one gets :

$$
\mathbb{L}: \boldsymbol{\theta}=\left[3 k \theta_{m}+2 \gamma\left(\boldsymbol{\theta}^{d}: \widehat{\boldsymbol{e}}\right)\right] \boldsymbol{i}+2 \mu \boldsymbol{\theta}^{d}+6 \gamma \theta_{m} \widehat{\boldsymbol{e}}+\frac{4}{3}(\lambda-\mu)\left(\boldsymbol{\theta}^{d}: \widehat{\boldsymbol{e}}\right) \widehat{\boldsymbol{e}}
$$

The equation $\mathbb{L}: \boldsymbol{\theta}=0$ reduces to two equations (hydrostatic and deviatoric part respectively) :

$$
3 k \theta_{m}+2 \gamma\left(\boldsymbol{\theta}^{d}: \widehat{\boldsymbol{e}}\right)=0, \quad 2 \mu \boldsymbol{\theta}^{d}+6 \gamma \theta_{m} \widehat{\boldsymbol{e}}+\frac{4}{3}(\lambda-\mu)\left(\boldsymbol{\theta}^{d}: \widehat{\boldsymbol{e}}\right) \widehat{\boldsymbol{e}}=0 .
$$

Since $\mu \neq 0$, the second equation shows that $\boldsymbol{\theta}^{d}$ is parallel to $\widehat{\boldsymbol{e}}$. Therefore $\boldsymbol{\theta}$ is in the form $\boldsymbol{\theta}=\theta_{m} \boldsymbol{i}+\alpha \widehat{\boldsymbol{e}}$ and the above system reduces to two scalar equations :

$$
k \theta_{m}+\gamma \alpha=0, \quad 3 \gamma \theta_{m}+\lambda \alpha=0
$$


This is a $2 \times 2$ homogeneous system of equations for the two unknowns $\left(\theta_{m}, \alpha\right)$. The linear space of solutions of this homogeneous system cannot be of dimension 2 since the coefficients $k, \gamma$ and $\lambda$ are not all equal to 0 . It cannot be of dimension 0 since, from the fact that $w$ is positively homogeneous of degree 1 it follows $\varepsilon: \mathbb{L}: \varepsilon=0$, and therefore $\left(\varepsilon_{m}, \varepsilon_{e q}\right)$ is a solution of this system. The only remaining option is that the space of solutions of (5.9) is of dimension 1. As a consequence, there exists $\Lambda \in \mathbb{R}$ such that $\theta_{m}=\Lambda \varepsilon_{m}$ et $\alpha=\Lambda \varepsilon_{e q}$, or in other words $\boldsymbol{\theta}=\Lambda \boldsymbol{\varepsilon}$. Therefore every element $\boldsymbol{\theta}$ in $\mathcal{L}_{\text {min }}$ is in the form $\boldsymbol{\theta}=\Lambda \boldsymbol{\varepsilon}$. This completes the proof of lemma 4 .

Proof of Theorem 1 : Let us first prove that if $\boldsymbol{\varepsilon}$ is rank-one, there exists $\boldsymbol{\xi}$ such that $\operatorname{det}(\mathbf{K}(\boldsymbol{\xi}))=0$. Since $\boldsymbol{\varepsilon}=\mathbf{a} \otimes_{s} \mathbf{b}$ with two non-vanishing vectors $\mathbf{a}$ and $\mathbf{b}$ and since $\boldsymbol{\varepsilon}$ is in $\mathcal{L}_{\text {min }}$, one has, $\mathbb{L}(\boldsymbol{\varepsilon}): \boldsymbol{\varepsilon}=\mathbb{L}(\varepsilon): \mathbf{a} \otimes_{s} \mathbf{b}=0$. Given the symmetries of $\mathbb{L}(\varepsilon)$, this relation can be alternatively written as :

$$
\mathbb{L}(\varepsilon) \cdot \mathbf{a} \cdot \mathbf{b}=0,
$$

and upon multiplication by a it becomes :

$$
\mathbf{a} \cdot \mathbb{L}(\varepsilon) \cdot \mathbf{a} \cdot \mathbf{b}=0 .
$$

Since $\mathbf{b} \neq 0$, one has $\operatorname{det}(\mathbf{a} \cdot \mathbb{L}(\boldsymbol{\varepsilon}) \cdot \mathbf{a})=0$. The acoustic tensor $\mathbf{K}$ evaluated at $\boldsymbol{\xi}=\mathbf{a} /\|\mathbf{a}\|$ is singular.

Conversely, let $\boldsymbol{\xi}$ be a vector for which $\operatorname{det}(\boldsymbol{\xi} \cdot \mathbb{L}(\boldsymbol{\varepsilon}) \cdot \boldsymbol{\xi})=0$. There exists a non-vanishing vector a such that $\boldsymbol{\xi} \cdot \mathbb{L}(\boldsymbol{\varepsilon}) \cdot \boldsymbol{\xi} \cdot \mathbf{a}=0$ and consequently

$$
\text { a. } \boldsymbol{\xi} \cdot \mathbb{L}(\varepsilon) \cdot \boldsymbol{\xi} \cdot \mathbf{a}=0
$$

By virtue of the symmetries exhibited by $\mathbb{L}(\varepsilon)$, this relation can be re-written as :

$$
\boldsymbol{\xi} \otimes_{s} \mathbf{a}: \mathbb{L}(\boldsymbol{\varepsilon}): \boldsymbol{\xi} \otimes_{s} \mathbf{a}=0 .
$$

Therefore $\boldsymbol{\xi} \otimes_{s}$ a belongs to $\mathcal{L}_{\text {min }}$. According to lemma $4, \boldsymbol{\xi} \otimes_{s}$ a is proportional to $\varepsilon$ and there exists $\Lambda$ such that $\varepsilon=\Lambda \boldsymbol{\xi} \otimes_{s} \mathbf{a}$. This proves that $\varepsilon$ is a rank-one symmetric tensor.

Remark: According to theorem 1, all strains $\varepsilon=\varepsilon_{e q}(T \boldsymbol{i}+\widehat{\boldsymbol{e}})$ for which there exists $\boldsymbol{\xi}$ such that $\operatorname{det}(\boldsymbol{\xi} . \mathbb{L}(\boldsymbol{\varepsilon}) \cdot \boldsymbol{\xi})=0$ are rank-one tensors. According to lemma 3 they are such that $|T| \leq 1 / 2$ and their eigenvalues, which can be written as $\varepsilon_{1}=\varepsilon_{e q}\left(T+\widehat{e}_{1}\right), \quad \varepsilon_{2}=\varepsilon_{e q}\left(T+\widehat{e}_{2}\right), \quad \varepsilon_{3}=\varepsilon_{e q}\left(T+\widehat{e}_{3}\right)$, satisfy $\varepsilon_{1} \leq \varepsilon_{2}=0 \leq \varepsilon_{3}$, (for $\omega \in\left[0, \frac{\pi}{3}\right]$ ). The condition $\varepsilon_{2}=0$ gives :

$$
T+\widehat{e}_{2}=T-\cos \left(\omega+\frac{\pi}{3}\right)=0
$$


In conclusion, $\Delta=\operatorname{det}(\mathbf{K}(\boldsymbol{\xi}))$ vanishes (and therefore $\delta$ is singular) when

$$
|T| \leq 1 / 2 \quad \text { and } \quad T=\cos \left(\omega+\frac{\pi}{3}\right)
$$

\subsection{The viscous case $m>0$}

In the viscous case $(m>0)$, the energy function (2.7) is strictly convex. Indeed, the function $\varepsilon \rightarrow\left(\frac{4 \varepsilon_{m}^{2}}{y^{2}}+\varepsilon_{e q}^{2}\right)^{\frac{m+1}{2}}$ is the composition of two functions : $x \rightarrow x^{m+1}$ and $\varepsilon \rightarrow\left(\frac{4 \varepsilon_{m}^{2}}{y^{2}}+\varepsilon_{e q}^{2}\right)^{\frac{1}{2}}$. The first one is an increasing strictly convex function for $m \neq 0$. The second function is convex (it behaves as the euclidean norm) but not strictly convex since its restriction to spherical tensors is a linear function. The result is a strictly convex function. Then, since the sum (or the integral) of strictly convex functions is strictly convex, the potential (2.7) is strictly convex for $m>0$ (it is convex, but not strictly convex, for $m=0$ ).

The strict convexity of $\widetilde{w}$ is not a sufficient for the positive definiteness of its second derivative. Consider for instance the function $x \rightarrow x^{4}$ which is strictly convex on $\mathbb{R}$ but whose second derivative vanishes at $x=0$. A direct proof of the positive definiteness of $\mathbb{L}=\frac{\partial^{2} \widetilde{w}}{\partial \varepsilon^{2}}$ will be given in the case of interest here. Note that the positive definiteness of $\mathbb{L}$ imply the positive definiteness of the acoustic tensor $\mathbf{K}(\boldsymbol{\xi})$. Indeed, if $\mathbb{L}$ is positive definite, it is in particular strictly positive on the rank-one tensors : $\mathbf{a} \otimes \boldsymbol{\xi}: \mathbb{L}: \boldsymbol{\xi} \otimes \mathbf{a}>0, \forall \mathbf{a}, \boldsymbol{\xi} \neq 0$ and therefore the acoustic tensor $\mathbf{K}(\boldsymbol{\xi})$ is invertible.

Lemma 5 Let $\mathbb{L}$ be a positive symmetric fourth-order tensor which can expressed as :

$$
\mathbb{L}=3 k \mathbb{J}+2 \lambda \mathbb{E}+2 \mu \mathbb{F}+2 \gamma \mathbb{G}
$$

and such that:

$$
k>0, \quad \lambda>0, \quad \mu>0, \quad k \lambda-3 \gamma^{2}>0 .
$$

Then $\mathbb{L}$ is positive definite on $\mathbb{R}_{s}^{3 \times 3}$.

Proof of lemma 5: Since $\mathbb{L}(\varepsilon)$ is positive and $\mu>0$ it follows, from the proof of lemma 4 :

$\mathcal{L}_{\text {min }}=\operatorname{Ker}(\mathbb{L})=\{\boldsymbol{\theta} \mid \mathbb{L}: \boldsymbol{\theta}=0\}=\left\{\boldsymbol{\theta}=\theta_{m} \boldsymbol{i}+\alpha \widehat{\boldsymbol{e}} \mid k \theta_{m}+\gamma \alpha=0,3 \gamma \theta_{m}+\lambda \alpha=0\right\}$.

The inequality $k \lambda-3 \gamma^{2}>0$ shows that the determinant of the homogeneous system for $\left(\theta_{m}, \alpha\right)$ does not vanish. Therefore the system has only one solution $:\left(\theta_{m}=0, \alpha=0\right)$. Consequently $\mathcal{L}_{\text {min }}=\{0\}$, which completes the proof of lemma 5 . 
Lemma 6 When the energy $\widetilde{w}$ is given by (2.7) with $m>0$, the coefficients (B.1) $k, \mu, \lambda$ and $\gamma$ of the second derivative of $\widetilde{w}$ satisfy the inequalities (5.10).

The proof of this rather technical lemma is given in appendix E. It follows from lemma 5 and 6 that the tangent moduli $\mathbb{L}$ associated with the energy (2.7) are positive definite when $m>0$.

\section{Perturbation of flow surfaces}

The effect of the local fluctuations in volume-fraction can be visualized on the flow surfaces (or equipotential surfaces) associated with the mesoscopic and macroscopic potentials $\widetilde{w}$ and $\widetilde{\widetilde{w}}$. The definition of a flow surface for rigidplastic and viscous materials is recalled in section 6.1 independently of any reference to composites. Then it is applied in section 6.2 to porous materials with local fluctuations in void-volume fraction.

\subsection{Flow surface}

Consider a power-law viscous materials characterized by a convex energy function $w$ which is a positively homogeneous function of degree $m+1$ with respect to $\varepsilon$. After suitable normalization $w$ can be written as

$$
w(\varepsilon)=\frac{1}{m+1} \frac{\sigma_{0}}{\varepsilon_{0}^{m}} w_{0}(\varepsilon),
$$

where $w_{0}$ is now a dimensionless convex and positively homogeneous function with degree $m+1$.

The flow surface of power-law materials is defined as an equipotential surface (or gauge surface in the terminology of Leblond et al. (1994)) for the potential $u$, convex dual of $w$ :

$$
\mathcal{S}=\left\{\boldsymbol{\sigma} \mid u(\boldsymbol{\sigma})=\frac{\varepsilon_{0}}{(n+1) \sigma_{0}^{n}}\right\}, \quad n=1 / m,
$$

where

$$
u(\boldsymbol{\sigma})=\sup _{\varepsilon}(\boldsymbol{\sigma}: \boldsymbol{\varepsilon}-w(\boldsymbol{\varepsilon})) .
$$

Since the potential $w$ is positively homogeneous of degree $m+1$ with respect to $\varepsilon$, its conjugate potential $u$ is a positively homogeneous function of degree $n+1$ (with $n=1 / m$ ) with respect to $\boldsymbol{\sigma}$. Therefore, for any stress state $\boldsymbol{\sigma}$, there exists a scalar $\Lambda(\boldsymbol{\sigma})$ called the gauge factor such that $\boldsymbol{\sigma} / \Lambda(\boldsymbol{\sigma}) \in \mathcal{S}$. From the 
definition (6.2) of the conjugate potential and from the positive homogeneity of $w$ we get the following equation for the gauge factor:

$$
\Lambda(\boldsymbol{\sigma})^{n+1} \frac{\varepsilon_{0}}{(n+1) \sigma_{0}^{n}}=u(\boldsymbol{\sigma})=\frac{1}{n} w(\boldsymbol{\varepsilon}) \quad \text { where } \boldsymbol{\varepsilon}=\frac{\partial u}{\partial \boldsymbol{\sigma}}(\boldsymbol{\sigma}) .
$$

The flow surface (6.1) can alternatively be determined from the potential $w$ (instead of $u$ ) by considering for each possible strain $\varepsilon$ a stress state $\boldsymbol{\sigma}^{\prime}$ on $\mathcal{S}$ defined as

$$
\boldsymbol{\sigma}^{\prime}=\frac{\boldsymbol{\sigma}}{\Lambda(\boldsymbol{\sigma})}, \quad \boldsymbol{\sigma}=\frac{\partial w}{\partial \boldsymbol{\varepsilon}}(\boldsymbol{\varepsilon}), \quad \Lambda(\boldsymbol{\sigma})=\left[\frac{(n+1) \sigma_{0}^{n}}{n \varepsilon_{0}} w(\overline{\boldsymbol{\varepsilon}})\right]^{\frac{1}{n+1}}
$$

For rigid-plastic materials $(m=0)$ the potential $w$ is positively homogeneous of degree one with respect to $\varepsilon$. Therefore the conjugate potential $u$ is the indicator function of the set of physically admissible stress states. A system of parametric equations for the flow surface can be obtained by letting $n$ go to $+\infty$ in $(6.3)$ :

$$
\boldsymbol{\sigma}^{\prime}=\frac{\boldsymbol{\sigma}}{\sigma_{0}}, \quad \boldsymbol{\sigma}=\frac{\partial w}{\partial \varepsilon}(\varepsilon)
$$

\subsection{Flow surfaces for porous materials with local fluctuations in void-volume fraction}

The above general definitions are now applied to define the flow surfaces $\widetilde{\mathcal{S}}$ and $\widetilde{\mathcal{S}}$ associated through the relations (6.1) or equivalently (6.3) to $\widetilde{w}$ given by $(2.7)$ and $\widetilde{w}$ respectively.

The dual potentials $\widetilde{u}$ and $\widetilde{\widetilde{u}}(2.7)$ are not known in closed form. It is therefore more convenient to use the characterization (6.3). We would like to use isotropy at the meso and macro-scale to further reduce to scalar equations the tensorial equations expressing the conjugacy relations :

$$
\boldsymbol{\sigma}=\frac{\partial \widetilde{w}}{\partial \varepsilon}(\varepsilon) \text { and } \quad \overline{\boldsymbol{\sigma}}=\frac{\partial \widetilde{\widetilde{w}}}{\partial \bar{\varepsilon}}(\bar{\varepsilon})
$$

This is can be easily achieved for $\widetilde{w}$ which depends only on the first two invariant of $\varepsilon$. The conjugacy relations reduce to

$$
\sigma_{m}=\frac{1}{3} \frac{\partial \widetilde{w}}{\partial \varepsilon_{m}}\left(\varepsilon_{m}, \varepsilon_{e q}\right), \quad \sigma_{e q}=\frac{\partial \widetilde{w}}{\partial \varepsilon_{e q}}\left(\varepsilon_{m}, \varepsilon_{e q}\right) .
$$

In contrast, the effective potential $\widetilde{\widetilde{w}}$ has an additional dependence on the angle $\omega$ related to the third invariant of $\bar{\varepsilon}$. The macroscopic conjugacy relations read 
as :

$$
\overline{\boldsymbol{\sigma}}=\frac{\partial \widetilde{\widetilde{w}}}{\partial \bar{\varepsilon}}\left(\bar{\varepsilon}_{m}, \bar{\varepsilon}_{e q}, \omega\right)=\frac{1}{3} \frac{\partial \widetilde{\widetilde{w}}}{\partial \bar{\varepsilon}_{m}} \boldsymbol{i}+\frac{2}{3} \frac{\partial \widetilde{\widetilde{w}}}{\partial \bar{\varepsilon}_{e q}} \widehat{\mathbf{e}}+\frac{\partial \widetilde{\widetilde{w}}}{\partial \omega} \frac{\partial \omega}{\partial \bar{\varepsilon}} .
$$

The last term in this relation introduces a complication in the calculation of the first two invariants of the macroscopic stress. To better understand this term we establish a few properties of $\omega$ as a function of $\bar{\varepsilon}$.

Lemma 7 The function $\omega(\overline{\boldsymbol{\varepsilon}})$ has the following properties : a) $\omega(\overline{\boldsymbol{\varepsilon}})$ is pressureinsensitive. In other words,

$$
\omega(\bar{\varepsilon}+\lambda \boldsymbol{i})=\omega(\bar{\varepsilon}) .
$$

b) $\omega(\overline{\boldsymbol{\varepsilon}})$ is a positively homogeneous function of degree 0 with respect to $\overline{\boldsymbol{\varepsilon}}$. In other words, for every positive $\lambda$

$$
\omega(\lambda \bar{\varepsilon})=\omega(\bar{\varepsilon}) .
$$

c) $\omega(\bar{\varepsilon})$ is an isotropic scalar function.

Proof of lemma \%: Points a) and b) result from the fact that $\omega$ depends on $\widehat{\mathbf{e}}$ only which depends neither on the hydrostatic part of $\bar{\varepsilon}$, nor on its von Mises norm. Point c) results from the fact that $\omega$ depends only on the eigenvalues and invariants of $\bar{\varepsilon}$ which are frame-independent quantities.

It follows from Lemma 7 that :

$$
\frac{\partial \omega}{\partial \bar{\varepsilon}}: \boldsymbol{i}=0, \quad \frac{\partial \omega}{\partial \bar{\varepsilon}}: \bar{\varepsilon}=0
$$

It follows from the first equation in (6.8) that $\partial \omega / \partial \overline{\boldsymbol{\varepsilon}}$ is deviatoric. Since $\widehat{\mathbf{e}}$ is deviatoric as well, the hydrostatic part of the macroscopic stress can then be identified from (6.5) :

$$
\bar{\sigma}_{m}=\frac{1}{3} \frac{\partial \widetilde{\widetilde{w}}}{\partial \bar{\varepsilon}_{m}}
$$

\subsubsection{Axisymmetric deformations}

For simplicity, we shall now restrict our attention to axisymmetric strains $(\omega=0)$. Since $\omega(\overline{\boldsymbol{\varepsilon}})$ is an isotropic function, its derivative $\boldsymbol{g}=\partial \omega / \partial \overline{\boldsymbol{\varepsilon}}$ has the same principal directions as $\bar{\varepsilon}$. All directions contained in the plane orthogonal to the axis of symmetry are principal directions for $\bar{\varepsilon}$ and therefore for $\boldsymbol{g}$. It follows that $\boldsymbol{g}$ is also axisymmetric with the same axis of symmetry as $\bar{\varepsilon}$. In the principal directions of both tensors, it follows from the relations (6.8) that the eigenvalues $g_{i}$ and $\bar{\varepsilon}_{i}$ of $\boldsymbol{g}$ and $\overline{\boldsymbol{\varepsilon}}$ satisfy:

$$
g_{1}+g_{2}+g_{3}=0, \quad g_{1} \bar{\varepsilon}_{1}+g_{2} \bar{\varepsilon}_{2}+g_{3} \bar{\varepsilon}_{3}=0 .
$$


Since for axisymmetric deformations one has $g_{1}=g_{2}$ and $\bar{\varepsilon}_{1}=\bar{\varepsilon}_{2}$, the above relations imply ${ }^{5} g_{1}=g_{2}=g_{3}=0$. In other words

$$
\frac{\partial \omega}{\partial \bar{\varepsilon}}(\bar{\varepsilon})=\mathbf{0} \text { for all axisymmetric } \bar{\varepsilon} .
$$

Coming back to (6.5) it seen that for axisymmetric deformations the macroscopic or constitutive relations reduce to :

$$
\overline{\boldsymbol{\sigma}}=\frac{\partial \widetilde{\widetilde{w}}}{\partial \bar{\varepsilon}}\left(\bar{\varepsilon}_{m}, \bar{\varepsilon}_{e q}, 0\right)=\frac{1}{3} \frac{\partial \widetilde{\widetilde{w}}}{\partial \bar{\varepsilon}_{m}} \boldsymbol{i}+\frac{2}{3} \frac{\partial \widetilde{\widetilde{w}}}{\partial \bar{\varepsilon}_{e q}} \widehat{\mathbf{e}}
$$

Therefore, for axisymmetric deformations, one has :

$$
\bar{\sigma}_{m}=\frac{1}{3} \frac{\partial \widetilde{\widetilde{w}}}{\partial \bar{\varepsilon}_{m}}, \quad \bar{\sigma}_{e q}=\left|\frac{\partial \widetilde{\widetilde{w}}}{\partial \bar{\varepsilon}_{e q}}\right| .
$$

The macroscopic stress corresponding to an axisymmetric deformation is axisymmetric, as a result of the isotropy of $\widetilde{\widetilde{w}}$. The converse is also true by isotropy of $\widetilde{\tilde{u}}$. Therefore assuming that the deformation is axisymmetric is equivalent to assuming that the stress is axisymmetric.

Finally, it follows from the expansion (3.3) that, up to second-order in the volume-fraction fluctuations and for axisymmetric stress states :

$$
\begin{aligned}
& \bar{\sigma}_{m}=\frac{1}{3} \frac{\partial \widetilde{w}}{\partial \bar{\varepsilon}_{m}}+\frac{t^{2} \Delta f^{2}}{6 c^{(1)} c^{(2)}}\left[\frac{\partial^{3} \widetilde{w}}{\partial f^{2} \partial \bar{\varepsilon}_{m}}-\frac{\partial}{\partial \bar{\varepsilon}_{m}}(\boldsymbol{\tau}: \mathbf{P}: \boldsymbol{\tau})\right] . \\
& \bar{\sigma}_{e q}=\left|\frac{\partial \widetilde{w}}{\partial \bar{\varepsilon}_{e q}}+\frac{t^{2} \Delta f^{2}}{2 c^{(1)} c^{(2)}}\left[\frac{\partial^{3} \widetilde{w}}{\partial f^{2} \partial \bar{\varepsilon}_{e q}}-\frac{\partial}{\partial \bar{\varepsilon}_{e q}}(\boldsymbol{\tau}: \mathbf{P}: \boldsymbol{\tau})\right]\right| .
\end{aligned}
$$

Note that the relation (6.12) is valid for all values of $\omega$, whereas (6.13) is valid for $\omega=0$.

The relations (6.12) and (6.13) can be used to plot the section of the flow surface $\widetilde{\mathcal{S}}$ corresponding to axisymmetric stresses. $\widetilde{\widetilde{\mathcal{S}}}$ is compared with $\widetilde{\mathcal{S}}$ in figure 7 . The data used to generate the plots are $f=0.1, \Delta f / f=0.1, t=1$ and $c^{(1)}=c^{(2)}=1 / 2$. A few comments can be made:

1) The "perturbed" flow surfaces $\widetilde{\mathcal{S}}$ are always located inside the "unperturbed" flow surfaces $\widetilde{\mathcal{S}}$. This observation is the graphical translation of conjecture 1 in section 4 .

2) The flow surfaces are more sensitive to porosity perturbations at high triaxialities $\left(\bar{\sigma}_{e q} \simeq 0\right)$ than at low triaxialities $\left(\bar{\sigma}_{m} \simeq 0\right)$.

$\overline{5}$ when $\bar{\varepsilon}_{1} \neq \bar{\varepsilon}_{3}$. Note that $\omega$ is undetermined when $\bar{\varepsilon}_{1}=\bar{\varepsilon}_{1}=\bar{\varepsilon}_{3}$. 

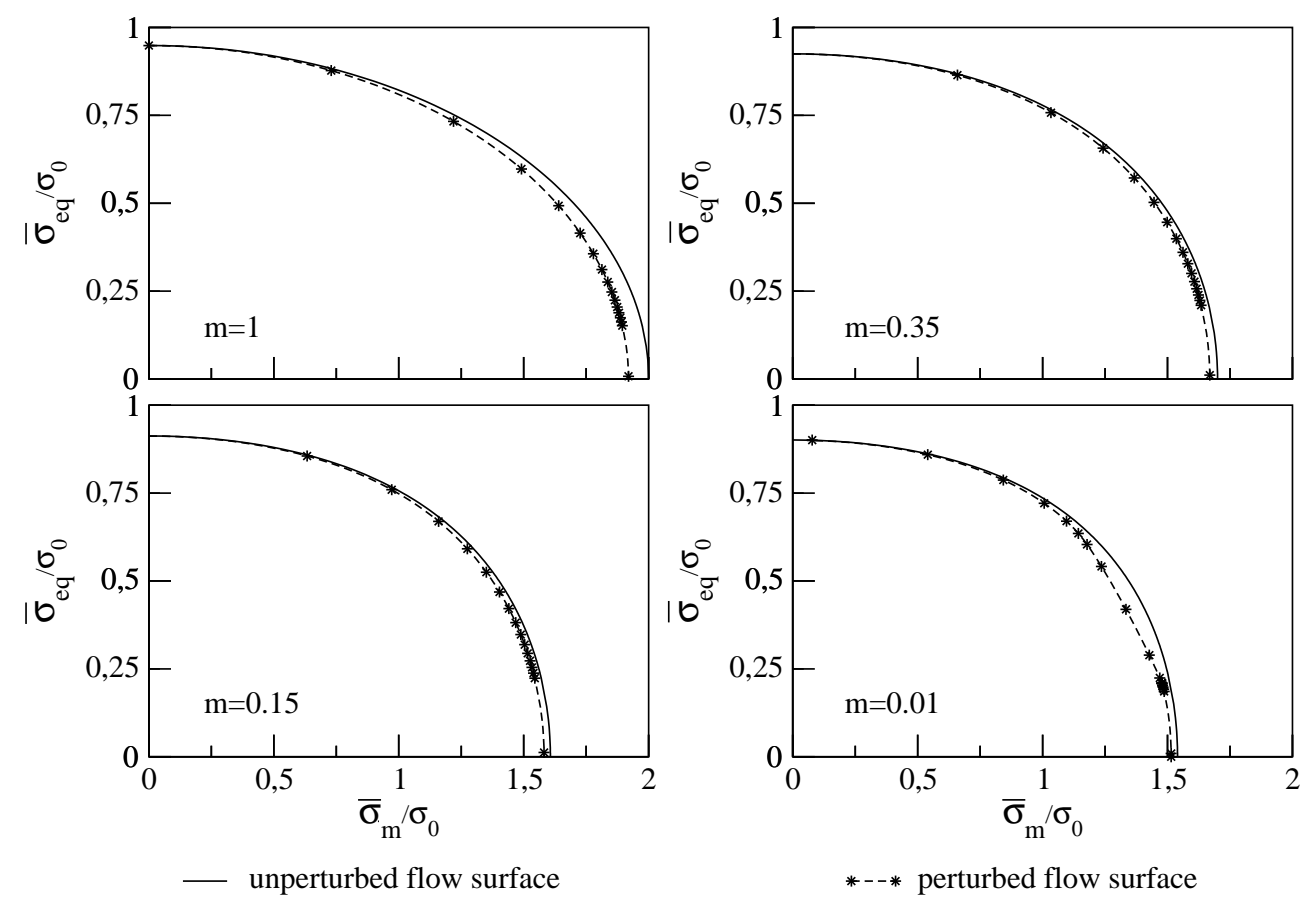

Fig. 7. Gauge surfaces

3) The surface corresponding to $m=0.01$ shows a slight change in convexity but still remains convex. Indeed, for small values of $m$ the second-order Taylor expansion $(3.3)$ of the potential $\widetilde{w}(\bar{\varepsilon})$ looses its convexity when the perturbation $\Delta f / f$ is too large. In the present case $(m=0.01)$ the value $\Delta f / f=0.1$ used to generate the plot approaches this critical value but is still below it. A larger porosity fluctuation would lead to a nonconvex flow surface. This uncorrect prediction stems from the fact that the expansion with respect to the fluctuations has been truncated to second order.

4) The plastic flow surface corresponding to the rigid-plastic case $(m=0)$ is not shown in the figure because the potential $\widetilde{\widetilde{w}}(\bar{\varepsilon})$ loses its convexity for any value of the perturbation $\Delta f / f$. This loss of convexity is due to the fact that $\delta$ is unbounded from below in the neighborhood of a rank-one deformation tensor (as discussed in section 5.1). In the axisymmetric case, accordingly to corollary 1 , there exists only one rank-one tensor $\bar{\varepsilon}$ which has the triaxiality-ratio $|T|=\frac{1}{2}$. This value of $T$ is related to the direction of the normal at the "stright" part of the gauge surface plotted in the figure for $m=0.01$. The potential $\widetilde{\widetilde{w}}(\bar{\varepsilon})$ is locally convex (for $\Delta f / f$ small enough) in the neighborhood of the points $\bar{\varepsilon}_{m}=0(T=0)$ and $\bar{\varepsilon}_{e q}=0 .(T=\infty)$.

\subsubsection{Rigid-plastic materials}

Gurson's yield function (2.5) is widely used to describe the effective yield surface of porous materials. How is it affected by local fluctuations in porosity? 
This question can be answered almost analytically for specific stress states. Indeed some of the terms in equations $(6.12-6.13)$ can be given in closed form:

$$
\begin{gathered}
\frac{\partial \widetilde{\widetilde{w}}}{\partial \bar{\varepsilon}_{m}}=-2\left[\log f+\log \frac{2 T+\sqrt{1+4 T^{2}}}{2 T+\sqrt{f^{2}+4 T^{2}}}\right], \quad \frac{\partial \widetilde{\widetilde{w}}}{\partial \bar{\varepsilon}_{e q}}=\sqrt{1+4 T^{2}}-\sqrt{f^{2}+4 T^{2}} \\
\frac{\partial^{3} \widetilde{\tilde{w}}}{\partial f^{2} \partial \bar{\varepsilon}_{m}}=\frac{8 T\left(f^{2}+2 T^{2}\right)}{f^{2}\left(f^{2}+4 T^{2}\right)^{3 / 2}}, \quad \frac{\partial^{3} \widetilde{\tilde{w}}}{\partial f^{2} \partial \bar{\varepsilon}_{e q}}=-\frac{4 T^{2}}{\left(f^{2}+4 T^{2}\right)^{3 / 2}}
\end{gathered}
$$

but the terms $\frac{\partial}{\partial \bar{\varepsilon}_{m}}(\boldsymbol{\tau}: \mathbf{P}: \boldsymbol{\tau})$ and $\frac{\partial}{\partial \bar{\varepsilon}_{e q}}(\boldsymbol{\tau}: \mathbf{P}: \boldsymbol{\tau})$ (which also involves an integration on the unit sphere) cannot be obtained analytically ${ }^{6}$. However, in the limiting cases $T=0$ and $T \rightarrow \infty$, these two derivatives simplify to

$$
\begin{aligned}
& \lim _{T \rightarrow 0} \frac{\partial(\boldsymbol{\tau}: \mathbf{P}: \boldsymbol{\tau})}{\partial \bar{\varepsilon}_{m}}=\frac{1}{\pi} \frac{1}{f(1+f)} \int_{\|\boldsymbol{\xi}\|=1} \frac{(\boldsymbol{\xi} . \widehat{\mathbf{e}} \cdot \boldsymbol{\xi})\left[\frac{1}{1-f}-\frac{4}{3}\left(\|\widehat{\mathbf{e}} \cdot \boldsymbol{\xi}\|^{2}-\frac{4+f}{4(1+f)}(\boldsymbol{\xi} . \widehat{\mathbf{e}} \cdot \boldsymbol{\xi})^{2}\right)\right]}{\left[1-\frac{4}{3}\left(\|\widehat{\mathbf{e}} \cdot \boldsymbol{\xi}\|^{2}-\frac{4+f}{4(1+f)}(\boldsymbol{\xi} . \widehat{\mathbf{e}} \cdot \boldsymbol{\xi})^{2}\right)\right]^{2}} \mathrm{~d} s(\boldsymbol{\xi}) \\
& \lim _{T \rightarrow 0} \frac{\partial(\boldsymbol{\tau}: \mathbf{P}: \boldsymbol{\tau})}{\partial \bar{\varepsilon}_{e q}}=\frac{1}{3 \pi} \frac{1}{1-f} \int_{\|\boldsymbol{\xi}\|=1} \frac{\|\widehat{\mathbf{e}} \cdot \boldsymbol{\xi}\|^{2}-\frac{4+f}{4(1+f)}(\boldsymbol{\xi} . \widehat{\mathbf{e}} \cdot \boldsymbol{\xi})^{2}}{1-\frac{4}{3}\left(\|\widehat{\mathbf{e}} \cdot \boldsymbol{\xi}\|^{2}-\frac{4+f}{4(1+f)}(\boldsymbol{\xi} . \widehat{\mathbf{e}} \cdot \boldsymbol{\xi})^{2}\right)}
\end{aligned}
$$

and

$$
\lim _{T \rightarrow \infty} \frac{\partial(\boldsymbol{\tau}: \mathbf{P}: \boldsymbol{\tau})}{\partial \bar{\varepsilon}_{m}}=\frac{4}{f^{2}\left(1-f^{2}\right)}, \quad \lim _{T \rightarrow \infty} \frac{\partial(\boldsymbol{\tau}: \mathbf{P}: \boldsymbol{\tau})}{\partial \bar{\varepsilon}_{e q}}=0 .
$$

Consequently, the corresponding values of $\bar{\sigma}_{m}$, denoted $\bar{\sigma}_{m}^{0}$ and $\bar{\sigma}_{m}^{\infty}$ respectively, read as:

$$
\frac{\bar{\sigma}_{m}^{0}}{\sigma_{0}}=-\frac{1}{6 \pi} \frac{\epsilon^{2} f}{c^{(1)} c^{(2)}(1+f)} \int_{\|\boldsymbol{\xi}\|=1} \frac{(\boldsymbol{\xi} . \hat{\mathbf{e}} \cdot \boldsymbol{\xi})\left[\frac{1}{1-f}-\frac{4}{3}\left(\|\widehat{\mathbf{e}} \cdot \boldsymbol{\xi}\|^{2}-\frac{4+f}{4(1+f)}(\boldsymbol{\xi} . \widehat{e} \cdot \boldsymbol{\xi})^{2}\right)\right]}{\left[1-\frac{4}{3}\left(\|\hat{\mathbf{e}} \cdot \boldsymbol{\xi}\|^{2}-\frac{4+f}{4(1+f)}(\boldsymbol{\xi} . \widehat{e} \cdot \boldsymbol{\xi})^{2}\right)\right]^{2}} \mathrm{~d} s(\boldsymbol{\xi})
$$

and

$$
\frac{\bar{\sigma}_{m}^{\infty}}{\sigma_{0}}=-\frac{2}{3} \log f+\frac{\epsilon^{2}}{3 c^{(1)} c^{(2)}}\left[1-\frac{2}{1-f^{2}}\right],
$$

respectively. And analogously :

$$
\begin{aligned}
\frac{\bar{\sigma}_{e q}^{0}}{\sigma_{0}} & \left.=\left|1-f-\frac{\epsilon^{2}}{c^{(1)} c^{(2)}} \frac{1}{6 \pi} \frac{f^{2}}{1-f} \int_{\|\boldsymbol{\xi}\|=1} \frac{\|\widehat{\mathbf{e}} \cdot \boldsymbol{\xi}\|^{2}-\frac{4+f}{4(1+f)}(\boldsymbol{\xi} . \widehat{\mathbf{e}} \cdot \boldsymbol{\xi})^{2}}{1-\frac{4}{3}\left(\|\widehat{\mathbf{e}} \cdot \boldsymbol{\xi}\|^{2}-\frac{4+f}{4(1+f)}(\boldsymbol{\xi} . \widehat{\mathbf{e}} \cdot \boldsymbol{\xi})^{2}\right)}\right| 16\right) \\
\frac{\bar{\sigma}_{e q}^{\infty}}{\sigma_{0}} & =0
\end{aligned}
$$

$\overline{6}$ The derivation inside the integral can be performed using Mathematica $^{\mathrm{TM}}$ 
Equations (6.14) and (6.15) are valid for any value of $\omega$, whereas (6.16) and $(6.17)^{7}$ are obtained from (6.13) which is valid only for $\omega=0$. Therefore relations (6.14) and (6.16) do not give a curve on the flow surface but only a point, that corresponding to $\omega=0$.

It is surprising at first that $\bar{\sigma}_{m}^{0}$ does not vanish (as could be expected). This stems from the dependence of the flow surface on the third invariant of the stress.

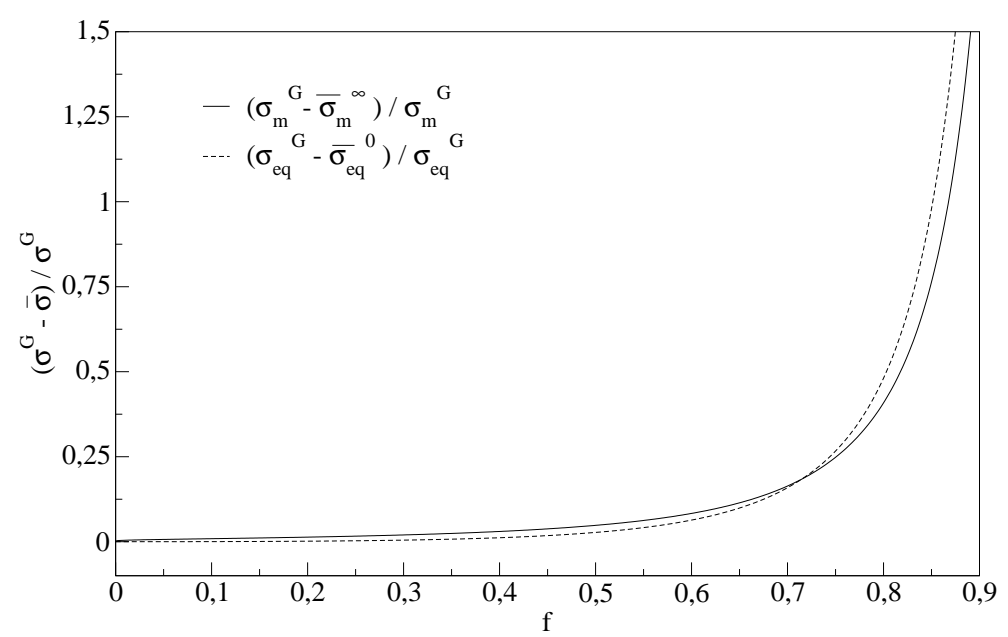

Fig. 8. Plots of the differences between the unperturbed (Gurson) and perturbed stresses $\bar{\sigma}_{m}$ and $\bar{\sigma}_{e q}$ as functions of $f$ for $\Delta f / f=0.1$ and $c^{(1)}=c^{(2)}=1 / 2$.

The question raised at the beginning of this section is answered in figure 8 where the differences between the stress $\boldsymbol{\sigma}^{G}$ in the unperturbed material (described by Gurson's model) and the stress $\overline{\boldsymbol{\sigma}}$ in the perturbed material are shown. Two specific cases, $T=0$ and $T \rightarrow+\infty$, are considered. The variations with the porosity $f$ of $\frac{\sigma_{m}^{G}}{\sigma_{0}}-\frac{\bar{\sigma}_{m}^{\infty}}{\sigma_{0}}$ and $\frac{\sigma_{e q}^{G}}{\sigma_{0}}-\frac{\bar{\sigma}_{e q}^{0}}{\sigma_{0}}$, where $\frac{\sigma_{m}^{G}}{\sigma_{0}}=-\frac{2}{3} \log f$, $\frac{\sigma_{e q}^{G}}{\sigma_{0}}=1-f$ are shown for $\Delta f / f=0.1$ and $c^{(1)}=c^{(2)}=1 / 2$. Several comments can be made.

1) As already mentioned, the perturbed stress are always smaller than the unperturbed ones.

2) The order of magnitude of the differences show that the flow surface is not much affected by porosity fluctuations. However, in the range of small to moderate porosities, the sensitivity of the Gurson flow surface at porosity fluctuation is greater at large triaxialities than at small triaxialities.

$\overline{7}$ In fact $\bar{\sigma}_{e q}^{\infty}=0$ for all values of $\omega$ since, for $T=\infty$, the macroscopic strain tensor $\bar{\varepsilon}$ is spherical and therefore $\bar{\sigma}$ is also spherical. 


\section{Conclusion}

The main findings of this study can be summarized as follows :

- First a general perturbation series expansion for the effective potential of composite materials with local fluctuations in the volume-fraction of their constituents has been derived (section 3).

- This expansion has been applied to nonlinear viscous or ideaaly plastic voided materials with a microstructure corresponding to the composite sphere assemblage (section 4).

- It has been observed numerically (section 4.5) and proved rigorously in a large range of strain triaxiality ratios or rate-sensitivity exponents (sections 4.3 and 4.4) that fluctuations in void-volume fraction always weaken the material. Therefore clusters of voids are likely to deteriorate the carrying capacity of materials containing microscopic voids.

- The effect of fluctuations is much more significant for purely hydrostatic loading conditions than for pure shear loading conditions (section 4.3). There are however intermediate strain triaxialities for which the effect of fluctuations can be even more pronounced.

- It has been proved (section 5) that the expansion becomes singular (and therefore breaks down) only in the special case of rigid-plastic materials and when the overall deformation is a rank-one deformation.

\section{Acknowledgments}

Part of this study has been carried out when M. Gărăjeu was a visiting research fellow at LMA. The financial support of CNRS is greatfully acknowledged. The authors wish to thank the refrees for their comments which helped in improving the manuscript. 


\section{References}

K. Bhattacharya. Microstructure of Martensite (why it forms and how it gives rise to the shape-memory effect). Oxford series on materials modelling. Oxford University Press, New York, 2003.

N. Bilger, F. Auslender, M. Bornert, J.C. Michel, H. Moulinec, P. Suquet, and A. Zaoui. Effect of a nonuniform distribution of voids on the plastic response of voided materials: a computational and statistical analysis. Int. J. Solids Structures, 42(2):517-538, 2005.

J.M. Duva and J.W. Hutchinson, Constitutive potentials for dilutely voided nonlinear materials, Mech. Materials, 3:41-54, 1984.

M. Gărăjeu, J.C. Michel, and P. Suquet. A micromechanical approach of damage in visco-plastic materials by evolution in size, shape and distribution of voids. Computer Methods in Applied Mechanics and Engineering, 183: 223-246, 2000.

A.L. Gurson. Continuum theory of ductile rupture by void nucleation and growth: Part i - yield criteria and flow rules for porous ductile media. $J$. Eng. Mat. Tech, 99:2-15, 1977.

L.M. Kachanov. Foundations of the Theory of Plasticity. North Holland, Amsterdam, 1971.

J.B. Leblond, G. Perrin, and P. Suquet. Exact results and approximate models for porous viscoplastic solids. International Journal of Plasticity, 10(3): 213-235, 1994.

G. Perrin. Contribution à l'étude théorique et numérique de la rupture ductile des métaux. PhD thesis, Ecole Polytechnique, 1992.

P. Ponte Castañeda. The effective mechanical properties of nonlinear isotropic composites. J. Mech. Phys. Solids, 39:45-71, 1991.

P. Ponte Castañeda. New variational principles in plasticity and their application to composite materials. J. Mech. Phys. Solids, 40(8):1757-1788, 1992.

P. Ponte Castañeda. Exact second-order estimates for the effective mechanical properties of nonlinear composite materials. J. Mech. Phys. Solids, 44(6): 827-862, 1996.

P. Ponte Castañeda and P. Suquet. Nonlinear composites. In E. Van der Giessen and T.Y. Wu, editors, Advances in Applied Mechanics, volume 34, pages 171-302. Academic Press, New York, 1998.

A.B. Richelsen and V. Tvergaard Dilatant plasticity or upper bounds estimates for porous ductile solids, Acta Metall. Mater., 422561-2577, 1994.

A. Rossoll, C. Berdin, and C. Prioul. Determination of the fracture toughness of a low alloy steel by the instrumented charpy impact test. Int. J. Fracture, 115(3):205 - 226, 2002.

J. Segurado, C. Gonzlez, and J. Llorca. A numerical investigation of the effect of particle clustering on the mechanical properties of composites. Acta Materialia, 51(8):2355-2369, 2003.

P. Suquet. On bounds for the overall potential of power law materials con- 
taining voids with an arbitrary shape. Mech. Res. Comm., 19(1):51-58, 1992.

P. Suquet. Overall potentials and extremal surfaces of power law or ideally plastic composites. J. Mech. Phys. Solids, 41:981-1002, 1993.

V. Tvergaard. Material failure by void growth to coalescence, volume 27 of Advances in Applied Mechanics. J.W. Hutchinson and T.Y. Wu, editors, Academic Press, New-York, 1990.

J. R. Willis. Variational and related methods for the overall properties of composites. In C.Y. Yih, editor, Advances in Applied Mechanics, volume 21, pages 1-78. Academic Press, New York, 1981.

J.R. Willis. On methods for bounding the overall properties of nonlinear composites. J. Mech. Phys. Solids, 39:73-86, 1991.

\section{A A preliminary result in linear algebra}

Lemma 8 Let $\boldsymbol{\xi}$ and $\boldsymbol{\eta}$ be two vectors in $\mathbb{R}^{3}, \lambda_{1}, \lambda_{2}, \lambda_{3}$ be 3 scalars and $\mathbf{K}$ be the matrix defined as

$$
\mathbf{K}=\boldsymbol{i}+\lambda_{1} \boldsymbol{\xi} \otimes \boldsymbol{\xi}+\lambda_{2}(\boldsymbol{\xi} \otimes \boldsymbol{\eta}+\boldsymbol{\eta} \otimes \boldsymbol{\xi})+\lambda_{3} \boldsymbol{\eta} \otimes \boldsymbol{\eta} .
$$

Then, whenever $\Delta=\operatorname{det}(\mathbf{K})$ is not equal to $0, \mathbf{K}$ is invertible and its inverse is given by:

$$
\mathbf{K}^{-1}=\boldsymbol{i}-\frac{1}{\Delta}\left[\Lambda_{1} \boldsymbol{\xi} \otimes \boldsymbol{\xi}+\Lambda_{2}(\boldsymbol{\xi} \otimes \boldsymbol{\eta}+\boldsymbol{\eta} \otimes \boldsymbol{\xi})+\Lambda_{3} \boldsymbol{\eta} \otimes \boldsymbol{\eta}\right]
$$

where

$$
\begin{aligned}
& \Lambda_{1}=\lambda_{1}+b^{2}\left(\lambda_{1} \lambda_{3}-\lambda_{2}^{2}\right), \quad \Lambda_{2}=\lambda_{2}-p\left(\lambda_{1} \lambda_{3}-\lambda_{2}^{2}\right), \quad \Lambda_{3}=\lambda_{3}+a^{2}\left(\lambda_{1} \lambda_{3}-\lambda_{2}^{2}\right), \\
& \left.\begin{array}{c}
\Delta=1+a^{2} \lambda_{1}+2 p \lambda_{2}+b^{2} \lambda_{3}+\left(\lambda_{1} \lambda_{3}-\lambda_{2}^{2}\right)\left(a^{2} b^{2}-p^{2}\right), \\
a=\|\boldsymbol{\xi}\|, \quad b=\|\boldsymbol{\eta}\|, \quad p=\boldsymbol{\xi} \cdot \boldsymbol{\eta} .
\end{array}\right\}
\end{aligned}
$$

Proof of lemma 8: It is sufficient to check that the product of $\mathbf{K}$ by (A.2) gives $\boldsymbol{i}$. This is easily done by using the relation $(\boldsymbol{u} \otimes \boldsymbol{v})(\boldsymbol{w} \otimes \boldsymbol{y})=(\boldsymbol{v} \cdot \boldsymbol{w}) \boldsymbol{u} \otimes \boldsymbol{y}$ for any vectors $\boldsymbol{u}, \boldsymbol{v}, \boldsymbol{w}, \boldsymbol{y} \in \mathbb{R}^{3}$.

The determinant of $\mathbf{K}$ is the product of its eigenvalues. Note that $\mathbf{K}$ can be written as 


$$
\begin{aligned}
\mathbf{K}=\boldsymbol{i} & +\left(\lambda_{1} a^{2}+2 p \lambda_{2}+\lambda_{3} \frac{p^{2}}{a^{2}}\right) \boldsymbol{u} \otimes \boldsymbol{u} \\
& +\sqrt{a^{2} b^{2}-p^{2}}\left(\lambda_{2}+\lambda_{3} \frac{p}{a^{2}}\right)(\boldsymbol{u} \otimes \boldsymbol{v}+\boldsymbol{v} \otimes \boldsymbol{u})+\lambda_{3} \frac{a^{2} b^{2}-p^{2}}{a^{2}} \boldsymbol{v} \otimes \boldsymbol{v}
\end{aligned}
$$

where

$$
\boldsymbol{u}=\frac{1}{a} \boldsymbol{\xi} \quad \text { and } \quad \boldsymbol{v}=\frac{a}{\sqrt{a^{2} b^{2}-p^{2}}}\left(\boldsymbol{\eta}-\frac{p}{a} \boldsymbol{u}\right) .
$$

The vectors $\boldsymbol{u}$ and $\boldsymbol{v}$ are two orthogonal unit vectors. The matrix representing $\mathbf{K}$ in the basis $(\boldsymbol{u}, \boldsymbol{v}, \boldsymbol{u} \wedge \boldsymbol{v})$, reads

$$
[\mathbf{K}]=\left[\begin{array}{ccc}
1+\lambda_{1} a^{2}+2 p \lambda_{2}+\lambda_{3} \frac{p^{2}}{a^{2}} & \sqrt{a^{2} b^{2}-p^{2}}\left(\lambda_{2}+\lambda_{3} \frac{p}{a^{2}}\right) & 0 \\
\sqrt{a^{2} b^{2}-p^{2}}\left(\lambda_{2}+\lambda_{3} \frac{p}{a^{2}}\right) & 1+\lambda_{3} \frac{a^{2} b^{2}-p^{2}}{a^{2}} & 0 \\
0 & 0 & 1
\end{array}\right] .
$$

Then, after elementary algebra, it follows that $\operatorname{det}(\mathbf{K})$ is given by the first relation in (A.3).

\section{B Detailed expressions for voided materials}

For porous materials whose effective behaviour is described by the Gursontype potential (2.7), the tensor $\mathbb{L}$ of instantaneous tangent moduli is given by (3.18) with :

$$
\begin{aligned}
& k=\frac{4 \sigma_{0}}{9 \varepsilon_{0}^{m}} \int_{f}^{1}\left(\frac{4 \bar{\varepsilon}_{m}^{2}}{y^{2}}+\bar{\varepsilon}_{e q}^{2}\right)^{\frac{m-3}{2}}\left(\frac{4 m \bar{\varepsilon}_{m}^{2}}{y^{2}}+\bar{\varepsilon}_{e q}^{2}\right) \frac{\mathrm{d} y}{y^{2}}=\frac{4 \sigma_{0}}{9 \varepsilon_{0}^{m}} \varepsilon_{e q}^{m-1}\left(\frac{1}{a(T, f)}-\frac{1}{a(T, 1)}\right), \\
& \mu=\frac{\sigma_{0}}{3 \varepsilon_{0}^{m}} \int_{f}^{1}\left(\frac{4 \bar{\varepsilon}_{m}^{2}}{y^{2}}+\bar{\varepsilon}_{e q}^{2}\right)^{\frac{m-1}{2}} \mathrm{~d} y=\frac{\sigma_{0}}{3 \varepsilon_{0}^{m}} \bar{\varepsilon}_{e q}^{m-1} \mathcal{I}_{m-1}, \\
& \lambda=\frac{\partial}{\partial \varepsilon_{e q}}\left(\varepsilon_{e q} \mu\right)=\frac{\sigma_{0}}{3 \varepsilon_{0}^{m}} \bar{\varepsilon}_{e q}^{m-1}\left[(m-1) \mathcal{I}_{m-3}+\mathcal{I}_{m-1}\right], \\
& \gamma=\frac{4 \sigma_{0}}{9 \varepsilon_{0}^{m}}(m-1) \bar{\varepsilon}_{e q} \bar{\varepsilon}_{m} \int_{f}^{1}\left(\frac{4 \bar{\varepsilon}_{m}^{2}}{y^{2}}+\bar{\varepsilon}_{e q}^{2}\right)^{\frac{m-3}{2}} \frac{\mathrm{d} y}{y^{2}}=\frac{\sigma_{0}(m-1)}{9 \varepsilon_{0}^{m}} \frac{\bar{\varepsilon}_{e q}^{m-1}}{T}\left(\mathcal{I}_{m-1}-\mathcal{I}_{m-3}\right)
\end{aligned}
$$


where

$$
\mathcal{I}_{k}=\int_{f}^{1}\left(\frac{4 T^{2}}{y^{2}}+1\right)^{\frac{k}{2}} \mathrm{~d} y, \quad T=\frac{\bar{\varepsilon}_{m}}{\bar{\varepsilon}_{e q}}, \quad a(T, f)=f^{m}\left(4 T^{2}+f^{2}\right)^{\frac{1-m}{2}} .
$$

The coefficients (3.19) of the acoustic tensor $\mathbf{K}(\boldsymbol{\xi})$ can be expressed as

$$
\begin{gathered}
\lambda_{1}=\frac{1}{3}+\frac{4}{3}\left[\frac{1}{a(T, f)}-\frac{1}{a(T, 1)}\right] \frac{1}{\mathcal{I}_{m-1}}, \quad \lambda_{2}=\frac{2}{3} \frac{m-1}{T}\left(1-\frac{\mathcal{I}_{m-3}}{\mathcal{I}_{m-1}}\right), \\
\lambda_{3}=\frac{4}{3}(m-1) \frac{\mathcal{I}_{m-3}}{\mathcal{I}_{m-1}}
\end{gathered}
$$

Furthermore :

$$
\frac{\partial^{2} \widetilde{w}}{\partial f^{2}}(f, \bar{\varepsilon})=\frac{4 \sigma_{0}}{\varepsilon_{0}^{m}} \frac{T^{2}}{f^{2} a(T, f)} \bar{\varepsilon}_{e q}^{m+1} .
$$

The components of the polarization stress $\boldsymbol{\tau}(3.20)$ are given by :

$$
\begin{aligned}
\tau_{m} & =\frac{1}{3} \frac{\partial^{2} \widetilde{w}}{\partial f \partial \varepsilon_{m}}(f, \bar{\varepsilon})=-\frac{4 \sigma_{0}}{3 \varepsilon_{0}^{m}} \frac{\bar{\varepsilon}_{m}}{f^{2}}\left(\frac{4 \bar{\varepsilon}_{m}^{2}}{f^{2}}+\bar{\varepsilon}_{e q}^{2}\right)^{\frac{m-1}{2}}=-\frac{4 \sigma_{0}}{3 \varepsilon_{0}^{m}} \bar{\varepsilon}_{e q}^{m} \frac{T}{f a(T, f)} \\
\tau_{e q} & =\frac{\partial^{2} \widetilde{w}}{\partial f \partial \varepsilon_{e q}}(f, \bar{\varepsilon})=-\frac{\sigma_{0}}{\varepsilon_{0}^{m}} \bar{\varepsilon}_{e q}\left(\frac{4 \bar{\varepsilon}_{m}^{2}}{f^{2}}+\bar{\varepsilon}_{e q}^{2}\right)^{\frac{m-1}{2}}=-\frac{\sigma_{0}}{\varepsilon_{0}^{m}} \bar{\varepsilon}_{e q}^{m} \frac{f}{a(T, f)} .
\end{aligned}
$$

\section{B.1 Pure dilatation}

When the macroscopic strain is a pure dilatation $\left(\bar{\varepsilon}_{e q}=0, T=\infty\right)$, the relations (B.1) give :

$$
\gamma=0, \quad k=\frac{4 \sigma_{0}}{9 \varepsilon_{0}^{m}}\left(2\left|\bar{\varepsilon}_{m}\right|\right)^{m-1}\left(\frac{1}{f^{m}}-1\right), \quad \lambda=\mu=\frac{\sigma_{0}}{3 \varepsilon_{0}^{m}}\left(2\left|\bar{\varepsilon}_{m}\right|\right)^{m-1} \frac{1-f^{2-m}}{2-m} .
$$

Therefore the tensors $\mathbb{L}$ and $\mathbb{P}$ are isotropic (the expression of $\mathbb{P}$ can be found in Willis (1981)) :

$$
\mathbb{L}=3 k \mathbb{J}+2 \mu \mathbb{K}, \quad \mathbb{P}=\frac{1}{3 k+4 \mu} \mathbb{J}+\frac{3(k+2 \mu)}{5 \mu(3 k+4 \mu)} \mathbb{K} .
$$

The polarization field $\boldsymbol{\tau}$ given by (B.4), reads here :

$$
\boldsymbol{\tau}=\tau_{m} \boldsymbol{i}=-\frac{4}{3} \frac{\sigma_{0}}{\varepsilon_{0}^{m}} \frac{\bar{\varepsilon}_{m}}{f^{m+1}}\left(2\left|\bar{\varepsilon}_{m}\right|\right)^{m-1} \boldsymbol{i} .
$$


Then:

$\boldsymbol{\tau}: \mathbb{P}: \boldsymbol{\tau}=\frac{\tau_{m}^{2}}{3 k+4 \mu} \boldsymbol{i}: \mathbb{I}: \boldsymbol{i}=\frac{3 \tau_{m}^{2}}{3 k+4 \mu}=\frac{\sigma_{0}}{\varepsilon_{0}^{m}}\left(\frac{2\left|\bar{\varepsilon}_{m}\right|}{f}\right)^{m+1} \frac{1}{f\left(1-f^{m}+\frac{f^{m}-f^{2}}{2-m}\right)}$

\section{B.2 Simple shear}

When the overall deformation is a simple shear $\left(\bar{\varepsilon}_{m}=0\right.$, i.e. $\left.T=0\right)$ relations (B.2) and (B.4) give

$$
\lambda_{1}=\frac{1}{3}+\frac{4}{3 f}, \quad \lambda_{2}=0, \quad \lambda_{3}=\frac{4}{3}(m-1)
$$

and

$$
\tau_{m}=0, \quad \tau_{e q}=-\frac{\sigma_{0}}{\varepsilon_{0}^{m}} \bar{\varepsilon}_{e q}^{m}
$$

Since $\tau_{m}=0$, it follows from (3.21) that :

$$
\boldsymbol{\tau}: \mathbb{P}: \boldsymbol{\tau}=\frac{1}{4 \pi} \int_{\|\xi\|=1} \frac{\frac{4}{9} \tau_{e q}^{2}\left[\left(1+\lambda_{1}\right) b^{2}-\lambda_{1} p^{2}\right]}{\mu \Delta} \mathrm{d} s(\boldsymbol{\xi})
$$

where $\mu$ is obtained from (B.1), $\mu=\frac{\sigma_{0}}{3 \varepsilon_{0}^{m}} \bar{\varepsilon}_{e q}^{m-1}(1-f)$ and $\Delta$ is given (with the notations of Appendix A) by $\Delta=\left(1+\lambda_{1}\right)\left[1+\lambda_{3}\left(b^{2}-\frac{\lambda_{1}}{1+\lambda_{1}} p^{2}\right)\right]$. Then

$$
\boldsymbol{\tau}: \mathbb{P}: \boldsymbol{\tau}=\frac{\sigma_{0}}{\varepsilon_{0}^{m}} \bar{\varepsilon}_{e q}^{m+1} \frac{1}{1-f} \frac{1}{3 \pi} \int_{\|\xi\|=1} \frac{b^{2}-\frac{\lambda_{1}}{1+\lambda_{1}} p^{2}}{1+\lambda_{3}\left(b^{2}-\frac{\lambda_{1}}{1+\lambda_{1}} p^{2}\right)} \mathrm{d} s(\boldsymbol{\xi})
$$

\section{Proof of Lemma 1}

$\Delta$ is a function of $\boldsymbol{\xi}$ on the unit sphere, $\|\boldsymbol{\xi}\|=\xi_{1}^{2}+\xi_{2}^{2}+\xi_{3}^{2}=1$ :

$$
\begin{gathered}
\Delta(\boldsymbol{\xi})=1+\lambda_{1}+2 p(\boldsymbol{\xi}) \lambda_{2}+b^{2}(\boldsymbol{\xi}) \lambda_{3}+\left(\lambda_{1} \Lambda_{3}-\lambda_{2}^{2}\right)\left(b^{2}(\boldsymbol{\xi})-p^{2}(\boldsymbol{\xi})\right) \\
p(\boldsymbol{\xi})=\boldsymbol{\xi} . \widehat{e} . \boldsymbol{\xi}, \quad b^{2}(\boldsymbol{\xi})=\boldsymbol{\xi} \cdot \widehat{\mathbf{e}}^{2} \cdot \boldsymbol{\xi}
\end{gathered}
$$

Then, the stationary points of $\Delta$ are the solutions of the following system :

$$
\left\{\begin{array}{l}
2 \lambda_{2} \widehat{e}_{i} \xi_{i}+\lambda_{3} \widehat{e}_{i}^{2} \xi_{i}+\left(\lambda_{1} \lambda_{3}-\lambda_{2}^{2}\right)\left(\widehat{e}_{i}^{2} \xi_{i}-2 p(\boldsymbol{\xi}) \widehat{e}_{i} \xi_{i}\right)-\Lambda \xi_{i}=0, \quad \text { for } i=1,2,3 \\
\xi_{1}^{2}+\xi_{2}^{2}+\xi_{3}^{2}=1
\end{array}\right.
$$

The solutions of this system lie on the circles $\xi_{1}=0, \xi_{2}=0$ or $\xi_{3}=0$. Indeed, if a solution is such that $\xi_{i} \neq 0$ for all $i=1,2,3$ then the eigenvalues 
$\left(\widehat{e}_{1}, \widehat{e}_{2}, \widehat{e}_{3}\right)$ of $\widehat{\mathbf{e}}$ are solutions of the second order equation :

$$
2 \lambda_{2} \widehat{e}_{i}+\lambda_{3} \widehat{e}_{i}^{2}+\left(\lambda_{1} \lambda_{3}-\lambda_{2}^{2}\right)\left(\widehat{e}_{i}^{2}-2 p(\boldsymbol{\xi}) \widehat{e}_{i}\right)-\Lambda=0 .
$$

The eigenvalues $\widehat{e}_{i}$ are all different $\left(\widehat{e}_{1}<\widehat{e}_{2}<\widehat{e}_{3}\right)$ if $\left.\omega \in\right] 0, \frac{\pi}{3}[$ (the case $\omega=0$ should be consider separately) and therefore the equation (C.1) has three different solutions. This is possible only if all the coefficients of the equation (C.1) vanish. In particular the coefficient of $\widehat{e}_{i}^{2}$ :

$$
\left(1+\lambda_{1}\right) \lambda_{3}-\lambda_{2}^{2}=0
$$

This equation can not be verified by $\lambda_{1}, \lambda_{2}$ and $\lambda_{3}$ since from (B.2) it follows $\lambda_{2} \neq 0, \lambda_{1}>0$ and $\lambda_{3}<0$, and therefore $\left(1+\lambda_{1}\right) \lambda_{3}-\lambda_{2}^{2}<0$.

Let $\Delta^{(i)}$ denotes the restriction of $\Delta$ to the circle $\xi_{i}=0$ (the other two components of $\boldsymbol{\xi}$ being $\cos \alpha$ and $\sin \alpha$ ). Straightforward algebra gives :

$$
\begin{aligned}
\Delta^{(1)}= & 1+\lambda_{1}+2 \widehat{e}_{2} \lambda_{2}+\widehat{e}_{2}^{2} \lambda_{3}-\left(\widehat{e}_{2}-\widehat{e}_{3}\right)\left[2 \lambda_{2}+\left(\widehat{e}_{2}-\widehat{e}_{3}\right)\left(\lambda_{2}^{2}-\lambda_{1} \lambda_{3}\right)+\right. \\
& \left.\left(\widehat{e}_{2}+\widehat{e}_{3}\right) \lambda_{3}\right] \sin ^{2} \alpha+\left(\widehat{e}_{2}-\widehat{e}_{3}\right)^{2}\left(\lambda_{2}^{2}-\lambda_{1} \lambda_{3}\right) \sin ^{4} \alpha
\end{aligned}
$$

and $\Delta^{(2)}$ and $\Delta^{(3)}$ are obtained by circular permutations on $\left(\widehat{e}_{1}, \widehat{e}_{2}, \widehat{e}_{3}\right)$. Since $\lambda_{2}^{2}-\lambda_{1} \lambda_{3}>0$ the coefficient of the dominant term is positive and therefore each of the function $\Delta^{(i)}$, reaches its maximum for $\sin \alpha=0$ or $\sin ^{2} \alpha=1$. The corresponding points on the unit sphere are $\boldsymbol{\xi}_{1}^{ \pm}=(0,1,0), \boldsymbol{\xi}_{2}^{ \pm}=(0,0,1)$ and $\boldsymbol{\xi}_{3}^{ \pm}=(0,0,1)$ Let $\Delta_{1}, \Delta_{2}$ and $\Delta_{3}$ denote the values of $\Delta$ at these points:

$$
\begin{aligned}
& \Delta_{1}=1+\lambda_{1}+2 \widehat{e}_{1} \lambda_{2}+\widehat{e}_{1}^{2} \lambda_{3}, \\
& \Delta_{2}=1+\lambda_{1}+2 \widehat{e}_{2} \lambda_{2}+\widehat{e}_{2}^{2} \lambda_{3}, \\
& \Delta_{3}=1+\lambda_{1}+2 \widehat{e}_{3} \lambda_{2}+\widehat{e}_{3}^{2} \lambda_{3} .
\end{aligned}
$$

Then, it follows:

$$
\Delta \leqslant \max \left(\Delta_{1}, \Delta_{2}, \Delta_{3}\right) .
$$

The strain components $\widehat{e}_{1}, \widehat{e}_{2}$ and $\widehat{e}_{3}$ are such that $\left|\widehat{e}_{i}\right| \leqslant 1$ and therefore

$$
\Delta \leqslant \max _{|x| \leqslant 1}\left(1+\lambda_{1}+2 x \lambda_{2}+x^{2} \lambda_{3}\right)
$$

Since $\lambda_{3}<0$, the function $x \rightarrow 1+\lambda_{1}+2 x \lambda_{2}+x^{2} \lambda_{3}$ has a maximum for $x=x_{\max }=-\frac{\lambda_{2}}{\lambda_{3}}$.

Using the expressions of the coefficients $\lambda_{2}$ and $\lambda_{3}$, in the case $m=0$ :

$$
\lambda_{2}=-\frac{8}{3} \frac{T}{A}, \quad \lambda_{3}=\frac{4}{3}\left(\frac{4 T^{2}}{A}-1\right), \quad A=a(T, 1) a(T, f) .
$$


one can show that $\left|x_{\max }\right| \geq 1$. Indeed, the following equivalences hold:

$$
\left|x_{\max }\right| \geq 1 \Leftrightarrow\left\{\begin{array}{l}
x_{\max } \leqslant-1 \Leftrightarrow \lambda_{2} \leqslant \lambda_{3} \Leftrightarrow 16 T^{3} \geqslant f^{2}\left(4 T^{2}+1\right), \quad \text { if } T \geq 0 \\
\text { or } \\
x_{\max } \geqslant 1 \Leftrightarrow \lambda_{2} \geqslant-\lambda_{3} \Leftrightarrow-16 T^{3} \geqslant f^{2}\left(4 T^{2}+1\right), \quad \text { if } T \leq 0
\end{array}\right.
$$

The function $h(x)=16 x^{3}-4 f^{2} x^{2}-f^{2}$ has a minimum at $x=\frac{f^{2}}{6}$ and is increasing when $x \geqslant \frac{f^{2}}{6}$. Therefore, when $|T| \geqslant \frac{1}{2}$, it follows that $16|T|^{3}-$ $4 f^{2} T^{2}-f^{2}=h(|T|) \geqslant h\left(\frac{1}{2}\right)=2\left(1-f^{2}\right) \geqslant 0$ and both inequalities in (C.4) are satisfied.

To complete the proof, note that:

- If $T \geq 0$ then $x_{\max } \leqslant-1$ and therefore $\Delta \leqslant 1+\lambda_{1}-2 \lambda_{2}+\lambda_{3}=\frac{4}{3 A}(1+2 T)^{2}$

- If $T \leq 0$ then $x_{\max } \geqslant 1$ and therefore $\Delta \leqslant 1+\lambda_{1}+2 \lambda_{2}+\lambda_{3}=\frac{4}{3 A}(1-2 T)^{2}$

and the lemma is proved.

\section{Proof of Lemma 2}

In order to prove that the function

$$
\begin{aligned}
f \rightarrow \mathcal{D}(f) & =T^{2}(1+2|T|)^{2}-\frac{3 a(T, f)^{2}}{20(1-B)}\left[1+\frac{2}{3} B+f^{2}\left(1-\frac{1}{B^{2}}\right)\right] \\
& =T^{2}(1+2|T|)^{2}+\left(\frac{4 T^{2}+1}{20}\right)\left(\frac{3 B^{2}+2 B^{3}+3 f^{2}\left(B^{2}-1\right)}{B-1}\right)
\end{aligned}
$$

is a decreasing function on $f$, note first that $\frac{\partial B}{\partial f}=\frac{f}{A}$, with $A=a(T, f) a(T, 1)$.

Then

$$
\begin{aligned}
\frac{\partial \mathcal{D}}{\partial f} & =\frac{\left(4 T^{2}+1\right) f}{20 A}\left[5+4 B+3 f^{2}+6 A(B+1)-\frac{5}{(1-B)^{2}}\right] \\
& =\frac{\left(4 T^{2}+1\right) f}{20 A(1-B)^{2}}\left[4 B^{3}-9 B^{2}+3 f^{2}(3 B-1)(B-1)\right]
\end{aligned}
$$

where the identity $\left(1-f^{2}\right) B=\left(1-B^{2}\right) A$ has been used. To complete the proof note that the last line can be written as:

$$
\frac{\partial \mathcal{D}}{\partial f}=-\frac{\left(4 T^{2}+1\right) f}{20 A(1-B)^{2}}\left[5 B^{2}+(1-B)\left(B^{2}+9 f^{2} B+3\left(B^{2}-f^{2}\right)\right)\right]<0 .
$$


The last inequality follows from the fact that $f \leqslant B \leqslant 1$.

\section{E Proof of Lemma 6}

The first three inequalities are direct consequences of the integral form of $k, \mu$ and $\lambda$. Note that the last inequality can be re-written as :

$$
\frac{\partial^{2} \widetilde{w}}{\partial \varepsilon_{m}^{2}}\left(\varepsilon_{m}, \varepsilon_{e q}\right) \frac{\partial^{2} \widetilde{w}}{\partial \varepsilon_{e q}^{2}}\left(\varepsilon_{m}, \varepsilon_{e q}\right)-\left(\frac{\partial^{2} \widetilde{w}}{\partial \varepsilon_{m} \partial \varepsilon_{e q}}\left(\varepsilon_{m}, \varepsilon_{e q}\right)\right)^{2}>0 \text { for every } \varepsilon_{m}, \varepsilon_{e q} .
$$

When the energy $\widetilde{w}$ takes the form $(2.7)$, straightforward calculations give :

$$
\begin{aligned}
\frac{\partial^{2} \widetilde{w}}{\partial \varepsilon_{m}^{2}}\left(\varepsilon_{m}, \varepsilon_{e q}\right) & =\frac{4 \sigma_{0}}{\varepsilon_{0}^{m}} \int_{f}^{1}\left(\frac{4 \bar{\varepsilon}_{m}^{2}}{y^{2}}+\bar{\varepsilon}_{e q}^{2}\right)^{\frac{m-3}{2}}\left(\frac{4 m \bar{\varepsilon}_{m}^{2}}{y^{2}}+\bar{\varepsilon}_{e q}^{2}\right) \frac{\mathrm{d} y}{y^{2}} \\
\frac{\partial^{2} \widetilde{w}}{\partial \varepsilon_{e q}^{2}}\left(\varepsilon_{m}, \varepsilon_{e q}\right) & =\frac{\sigma_{0}}{\varepsilon_{0}^{m}} \int_{f}^{1}\left(\frac{4 \bar{\varepsilon}_{m}^{2}}{y^{2}}+\bar{\varepsilon}_{e q}^{2}\right)^{\frac{m-3}{2}}\left(\frac{4 \bar{\varepsilon}_{m}^{2}}{y^{2}}+m \bar{\varepsilon}_{e q}^{2}\right) \mathrm{d} y \\
\frac{\partial^{2} \widetilde{w}}{\partial \varepsilon_{m} \partial \varepsilon_{e q}}\left(\varepsilon_{m}, \varepsilon_{e q}\right) & =\frac{4 \sigma_{0}}{\varepsilon_{0}^{m}} \bar{\varepsilon}_{m} \bar{\varepsilon}_{e q} \int_{f}^{1}\left(\frac{4 \bar{\varepsilon}_{m}^{2}}{y^{2}}+\bar{\varepsilon}_{e q}^{2}\right)^{\frac{m-3}{2}} \frac{\mathrm{d} y}{y^{2}}
\end{aligned}
$$

The desired inequality is equivalent to :

$$
\left(\int_{f}^{1} F^{2}(y) \mathrm{d} y\right)\left(\int_{f}^{1} G^{2}(y) \mathrm{d} y\right) \geq\left(\int_{f}^{1} H(y) \mathrm{d} y\right)^{2},
$$

where:

$$
\begin{gathered}
F(y)=\left(\frac{4 \bar{\varepsilon}_{m}^{2}}{y^{2}}+\bar{\varepsilon}_{e q}^{2}\right)^{\frac{m-3}{4}}\left(\frac{4 m \bar{\varepsilon}_{m}^{2}}{y^{2}}+\bar{\varepsilon}_{e q}^{2}\right)^{1 / 2} \frac{1}{y}, \quad G(y)=\left(\frac{4 \bar{\varepsilon}_{m}^{2}}{y^{2}}+\bar{\varepsilon}_{e q}^{2}\right)^{\frac{m-3}{4}}\left(\frac{4 \bar{\varepsilon}_{m}^{2}}{y^{2}}+m \bar{\varepsilon}_{e q}^{2}\right)^{1 / 2}, \\
H(y)=\left(\frac{4 \bar{\varepsilon}_{m}^{2}}{y^{2}}+\bar{\varepsilon}_{e q}^{2}\right)^{\frac{m-3}{2}} \frac{2}{y^{2}} \bar{\varepsilon}_{m} \bar{\varepsilon}_{e q} .
\end{gathered}
$$

According to Cauchy-Schwartz inequality, one has :

$$
\left(\int_{f}^{1} F^{2}(y) \mathrm{d} y\right)\left(\int_{f}^{1} G^{2}(y) \mathrm{d} y\right) \geq\left(\int_{f}^{1} F(y) G(y) \mathrm{d} y\right)^{2},
$$

with $F(y) G(y)=\left(\frac{4 \bar{\varepsilon}_{m}^{2}}{y^{2}}+\bar{\varepsilon}_{e q}^{2}\right)^{\frac{m-3}{2}}\left(\frac{16 m}{y^{4}} \bar{\varepsilon}_{m}^{4}+m \bar{\varepsilon}_{e q}^{4}+\frac{4}{y^{2}}\left(m^{2}+1\right) \bar{\varepsilon}_{m}^{2} \bar{\varepsilon}_{e q}^{2}\right)^{1 / 2} \frac{1}{y}$.

But

$$
\frac{16 m}{y^{4}} \bar{\varepsilon}_{m}^{4}+m \bar{\varepsilon}_{e q}^{4} \geq \frac{8 m}{y^{2}} \bar{\varepsilon}_{m}^{2} \bar{\varepsilon}_{e q}^{2}
$$


and therefore :

$$
F(y) G(y) \geq\left(\frac{4 \bar{\varepsilon}_{m}^{2}}{y^{2}}+\bar{\varepsilon}_{e q}^{2}\right)^{\frac{m-3}{2}} \frac{2(m+1)}{y^{2}} \bar{\varepsilon}_{m} \bar{\varepsilon}_{e q}>H(y) \quad \text { when } m>0
$$

This completes the proof of lemma 6 . 